\title{
Option PRices AND Risk- Neutral Densities for Currency Cross Rates
}

\section{STEPHEN J. TAYLOR YAW-HUEI WANG*}

\begin{abstract}
The theoretical relationship between the risk-neutral density (RND) of the euro/ pound cross rate and the bivariate RND of the dollar/euro and the dollar/pound rates is derived; the required bivariate RND is defined by the dollar-rate marginal RNDs and a copula function. The cross-rate RND can be used by banks, international businesses, and central bankers to assess market expectations, to measure risks, and to value options, without relying on over-the-counter markets, which may be either non-existent or illiquid. Empirical comparisons are made between cross-rate RNDs estimated from several data sets. Five one-parameter copula functions are evaluated and it is found that the Gaussian copula is the only oneparameter copula function that is ranked highly in all of the comparisons we have made. (C) 2009 Wiley Periodicals, Inc. Jrl Fut Mark 30:324-360, 2010
\end{abstract}

\footnotetext{
We thank the referee for the helpful advice. This research has benefited from numerous conversations with Söhnke Bartram, and the assistance of Aris Bikos with data collection is gratefully acknowledged. We are indebted to the seminar participants at the European Finance Association 2004 Annual Meeting, the Conference on the Theories and Practices of Securities and Financial Markets 2004, and the NTU International Conference on Finance 2004 for the helpful comments and suggestions.
}

*Correspondence author, Department of Finance, National Taiwan University, No. 1, Sec. 4, Roosevelt Road, Taipei 10617, Taiwan. Tel: +886-2-33661092, Fax: +886-2-83695581, e-mail: yhwang@management. ntu.edu.tw

Received June 2008; Accepted May 2009

- Stephen J. Taylor is a Professor of Finance at the Department of Accounting and Finance, Lancaster University, Lancaster, UK.

- Yaw-Huei Wang is an Associate Professor of Finance at the Department of Finance, National Taiwan University, Taipei, Taiwan. 


\section{INTRODUCTION}

Option markets provide important information about market expectations for foreign exchange rates. The future volatility of rates can be predicted by inverting a pricing formula and these predictions are known to be generally superior to forecasts obtained by estimating time series models from historical rates (Jorion, 1995; Pong, Shackleton, Taylor, \& Xu, 2004). Risk managers and policy makers use implied volatilities but they are also interested in the additional information provided by probability densities for future rates. In particular, the most prominent users of option-based density expectations are central banks (Bliss \& Panigirtzoglou, 2002; Söderlind \& Svensson, 1997).

Many numerical methods can be used to convert a set of option prices into a univariate risk-neutral density (RND) and these are routinely applied to dollar exchange rates (Campa, Chang, \& Reider, 1998; Jondeau \& Rockinger, 2000; Malz, 1996, 1997). A manager, a trader, or a central banker concerned about the euro/pound cross rate may find, however, that these univariate methods are infeasible. The estimation and analysis of RNDs for exchange cross rates is a difficult problem because cross-rate options have low liquidity and are traded over-the-counter (OTC), at prices that are not usually available to researchers. The contrast between the abundance of dollar option prices and the paucity of non-dollar option prices suggests that the most constructive way to learn about market expectations for cross rates may involve exploiting the triangular relationship between cross rates and a pair of dollar rates (Bennett \& Kennedy, 2004).

The primary contribution of this study is a complete methodology for estimating cross-rate RNDs from which cross-rate options can be priced. Our methods are illustrated for options on the euro/pound rate and they rely on prices from the highly liquid option markets for the dollar/pound and the dollar/euro rates. We are the first to use the triangular relationship between three currencies to infer the correct cross-rate RND from the bivariate density of the two dollar exchange rates. This bivariate density is given by the product of three terms: the two marginal densities for the dollar rates and a copula function that quantifies their dependence.

The second contribution of this study is advice about selecting the copula function, which is based upon comparing our derived cross-rate RNDs with those implied by OTC RNDs. The cross-rate RNDs that we derive can be used by banks, international businesses, and central bankers to assess market expectations, to measure risks, and to value options, without relying on OTC markets, which may be either non-existent or illiquid.

Bikos (2000), Rosenberg (2003), and Bennett and Kennedy (2004) also investigate cross-rate densities, their option prices, and the implied dependence 
between dollar exchange rates. Bikos (2000) uses option prices for all three exchange rates to estimate the dependence between dollar rates. Rosenberg (2003) shows how the bivariate density can be estimated non-parametrically, using a copula function that is estimated from historical exchange rates. As we explain in the following section, his cross-rate density is not risk-neutral because a required change of numeraire from dollars (for the bivariate density) to a foreign currency (for the cross-rate density) is overlooked. Bennett and Kennedy (2004) provide some related theoretical results for quanto options, which have dollar payoffs that are contingent upon cross rates.

Our empirical results are all for the dollar, pound, and euro currencies. We compare the option prices for cross rates quoted by a bank with those that we derive from dollar option prices. Our derived prices depend on a source, a copula function, and its dependence parameter. We compare the results for dollar option prices obtained from two sources: the OTC market and the Chicago Mercantile Exchange (CME). We compare results for five widely used copula functions, namely the Clayton, Frank, Gaussian, Gumbel, and Plackett copulas. For each copula function, we fix the dependence parameter by using either the at-the-money (ATM), OTC cross-rate price or the recent historical record of exchange rates. We find first that the publicly available CME source gives essentially the same results as the OTC source, second that the Frank, Gaussian, and Gumbel copulas provide the most satisfactory option prices, and third that historical correlations are on average lower than those implied by cross-rate, OTC option prices.

This article continues with three theoretical sections, followed by three empirical sections. The following section derives the relationship between the RND for the cross rate and the bivariate RND for two dollar exchange rates, using risk-neutral measures determined by the numeraires of payoffs. An introduction to copula functions is then provided; these are used to characterize the relationship between the bivariate RND and the marginal RNDs for the dollar rates. The third theoretical section summarizes three standard methods for estimating marginal RNDs. The empirical framework, the data, and the empirical results are then presented. Finally, conclusions are stated.

\section{THE RISK-NEUTRAL DENSITY FORMULA FOR CROSS RATES}

Our first objective is to produce formulae for the cross-rate RND and the prices of cross-rate options by using the prices of dollar-denominated assets. Option payoffs depend on the rates of exchange between dollars (\$, USD), pounds $(£, G B P)$, and euros (€, EUR) in our three-currency framework. We denote the dollar price of one pound at time $t$ by $S_{t}^{\$ / £}$ and likewise the dollar price of one 
euro at the same time is denoted by $S_{t}^{\$ / \epsilon}$. The cross-rate price of one pound in euros is then given by $S_{t}^{€ / £}=S_{t}^{\$ / \epsilon} / S_{t}^{\$ / €}$.

At time zero we assume that there is a complete market for $\$ / £$ European call options, priced in dollars, which expire at time $T$. This implies the existence of a unique RND for $S_{t}^{\$ / E}$ that we denote by $f_{\S}(y)$; the dollar subscript emphasizes that the numeraire of asset payoffs is dollars. Likewise, there is an RND for $S_{t}^{\$ / €}$ that we denote by $f_{\$}(z)$. We also assume that it is possible to define a bivariate RND for $\left(S_{t}^{\$ / €}, S_{T}^{\$ / €}\right)$, denoted by $f_{\S}(y, z)$, which can be used to price dollar payoffs that are contingent on these two exchange rates; we defer discussion of the construction of $f_{\S}(y, z)$ until the following section.

Now consider an European option to buy $£ 1$ for $€ X$ at time $T$. This is identical to an option to exchange $X S_{T}^{\$ / \mathcal{E}}$ dollars for $S_{T}^{\$ / \mathbb{E}}$ dollars at time $T$. Hence its dollar payoff equals $\max \left(S_{T}^{\$ / €}-X S_{T}^{\$ / €}, 0\right)$ and its fair price in dollars at time zero is

$$
\begin{aligned}
C_{\$}(X) & =e^{-r_{\S} T} E^{Q_{\S}}\left[\max \left(S_{T}^{\$ / €}-X S_{T}^{\$ / €}, 0\right)\right] \\
& =e^{-r_{\S} T} \int_{0}^{\infty} \int_{0}^{\infty} \max (y-X z, 0) f_{\$}(y, z) d y d z .
\end{aligned}
$$

Here $Q_{\$}$ is the risk-neutral measure for the $\$$ numeraire and $r_{\$}$ is the dollar risk-free rate. The fair price of the same option in euros is therefore

$$
\begin{aligned}
C_{€}(X) & =C_{\$}(X) / S_{0}^{\$ / €} \\
& =\left(e^{-r_{\S} T} / S_{0}^{\$ € €}\right) \int_{0}^{\infty} \int_{0}^{\infty} \max (y-X z, 0) f_{\$}(y, z) d y d z .
\end{aligned}
$$

This must equal the following discounted expected payoff, which employs the risk-neutral measure $Q_{€}$ for the euro numeraire:

$$
\begin{aligned}
C_{€}(X) & =e^{-r_{\epsilon} T} E^{Q_{\epsilon}}\left[\max \left(S_{T}^{€ / €}-X, 0\right)\right] \\
& =e^{-r_{\epsilon} T} \int_{X}^{\infty}(x-X) f_{€}(x) d x .
\end{aligned}
$$

Here $r_{€}$ is the euro risk-free rate and $f_{€}(x)$ is the RND for one pound priced in euros.

A specific formula for the cross-rate RND defined by Equation (3) is given by using the well-known result of Breeden and Litzenberger (1978):

$$
f_{€}(x)=e^{r_{\epsilon} T} \frac{\partial^{2} C_{€}(x)}{\partial x^{2}}
$$

in conjunction with Equation (2) to obtain 


$$
f_{€}(x)=\frac{1}{F_{0}^{\$ / €}} \frac{\partial^{2}}{\partial x^{2}}\left[\int_{0}^{\infty} \int_{0}^{\infty} \max (y-x z, 0) f_{\$}(y, z) d y d z\right]
$$

where $F_{0}^{\$ / €}=S_{0}^{\$ / €} e^{\left(r_{\$}-r_{€}\right) T}$ is the forward price, at time zero, to exchange one euro for dollars at time T. Equation (4) simplifies, as shown in Appendix A, to give the RND of the cross rate as the following single integral:

$$
f_{€}(x)=\frac{1}{F_{0}^{\$ / €}} \int_{0}^{\infty} z^{2} f_{\$}(x z, z) d z
$$

Cross-rate option prices can be calculated either from the two single integrals defined by Equations (3) and (5), or from the double integral in Equation (2), which is the same as

$$
C_{€}(X)=e^{-r_{€} T} \frac{1}{F_{0}^{\$ / €}} \int_{X}^{\infty}(x-X)\left[\int_{0}^{\infty} z^{2} f_{\$}(x z, z) d z\right] d x .
$$

It is easy to check the three necessary conditions for the function in (5) to be an RND. First, the function is obviously non-negative. Second, the substitution $y=x z$ (with $d y=z d x$ ) can be used to show that the function integrates to one and is therefore a density:

$$
\begin{aligned}
\int_{0}^{\infty} f_{€}(x) d x & =\frac{1}{F_{0}^{\$ / €}} \int_{0}^{\infty} z^{2}\left[\int_{0}^{\infty} f_{\$}(x z, z) d x\right] d z \\
& =\frac{1}{F_{0}^{\$ / €}} \int_{0}^{\infty} \int_{0}^{\infty} z f_{\$}(y, z) d y d z=E^{Q_{S}}\left[S_{T}^{\$ / €}\right] / F_{0}^{\$ / €}=1 .
\end{aligned}
$$

Third, the same substitution establishes that the expectation of the spot cross rate at time $T$ is the forward cross rate, and hence the density is risk-neutral:

$$
\begin{aligned}
\int_{0}^{\infty} x f_{€}(x) d x & =\frac{1}{F_{0}^{\$ / €}} \int_{0}^{\infty} \int_{0}^{\infty} x z^{2} f_{\$}(x z, z) d x d z \\
& =\frac{1}{F_{0}^{\$ / €}} \int_{0}^{\infty} \int_{0}^{\infty} y f_{\$}(y, z) d y d z \\
& =E^{Q_{\$}}\left[S_{T}^{\$ / €}\right] / F_{0}^{\$ / €}=F_{0}^{\$ / €} / F_{0}^{\$ / €}=F_{0}^{€ / €}
\end{aligned}
$$

We provide the above checks because our RND for the cross rate is not the same as the density derived in Rosenberg (2003). His density, in our notation, is

$$
f(x)=\int_{0}^{\infty} z f_{\$}(x z, z) d z
$$


This is the density of the cross rate with respect to the risk-neutral measure for the dollar numeraire, i.e. $Q_{\$}$. Thus, (9) is only appropriate for the valuation of claims that have dollar payoffs (e.g. the quanto options valued by Bennett \& Kennedy, 2004). Consequently, the Rosenberg density is not in general a crossrate RND and hence it should not be used to price cross-rate options. Indeed, a random variable whose density is given by (9) has expectation

$$
\begin{aligned}
\iint x z f_{\$}(x z, z) d z d x & =\iint \frac{y}{z} f_{\$}(y, z) d y d z \\
& =E^{Q_{s}}\left[\frac{S_{T}^{\$ / €}}{S_{T}^{\$ / €}}\right] \neq \frac{E^{Q_{\$}}\left[S_{T}^{\$ / €}\right]}{E^{Q_{s}}\left[S_{T}^{\$ / €}\right]}=\frac{F_{0}^{\$ / €}}{F_{0}^{\$ / €}}=F_{0}^{€ / €}
\end{aligned}
$$

whenever $\operatorname{Cov}^{Q \$}\left(S_{T}^{\$ / €}, S_{T}^{\$ / €} / S_{T}^{\$ / €}\right) \neq 0$.

\section{THE CONSTRUCTION OF BIVARIATE RNDS}

To price cross-rate options, using the cross-rate RND given by (5), we first need to obtain the bivariate RND of the two dollar-denominated exchange rates, denoted by $f_{\$}(y, z)$. We use copula functions in this article to convert the two marginal densities, $f_{\$}(y)$ and $f_{\$}(z)$, into the bivariate density $f_{\$}(y, z)$.

Copula methods are covered in the textbooks by Joe (1997), Nelsen (1999), and Cherubini, Luciano, and Vecchiato (2004). There are many recent applications in finance research to subjects such as credit risk ( $\mathrm{Li}, 2000)$, portfolio allocations (Hennessy \& Lapan, 2002), the pricing of multivariate contingent claims (Rosenberg, 2003), and the dependence between European markets (Bartram, Taylor, \& Wang, 2007).

\section{Definitions}

Copula functions, denoted $C(u, v)$, are the bivariate cumulative distribution functions (c.d.f.) of random variables $U$ and $V$ whose marginal distributions are uniform from zero to one. Employing a copula function permits modeling of dependence by using marginal densities to construct bivariate densities that are consistent with the univariate marginals.

Let $H$ be the bivariate c.d.f. for random variables $Y$ and $Z$, with respective marginal cumulative functions $F$ and $G$ that are both continuous. By Sklar's Theorem, there exists a unique copula function $C$ such that

$$
H(y, z)=C(F(y), G(z)) \text { for all } y \text { and } z \text {. }
$$


The bivariate density for $(Y, Z)$ is given by

$$
h(y, z)=c(F(y), G(z)) \times f(y) \times g(z)
$$

where $c(u, v)=\partial^{2} C(u, v) /(\partial u \partial v)$ is the density corresponding to the c.d.f. $C(u, v)$ and $f$ and $g$ are the marginal densities of $Y$ and $Z$.

The correlation between the uniform random variables $U=F(Y)$ and $V=$ $G(Z)$ is a useful parameter for measuring the dependence between $Y$ and $Z$. This correlation defines Spearman's " $\rho$ " for the variables $Y$ and $Z$, denoted by $\rho_{S}$ :

$$
\rho_{S}(Y, Z)=\operatorname{cor}(U, V)=\left(E[U V]-\frac{1}{4}\right) / \frac{1}{12} .
$$

Spearman's $\rho$ is obviously invariant to increasing transformations of $Y$ and Z. In particular, $\rho_{S}(Y, Z)=\rho_{S}(U, V)$.

\section{Specific Copula Functions}

Although several parametric copula functions have been applied in statistical literature, only a few of them have flexible dependence properties and are hence appropriate for financial applications (Joe, 1997). We focus on five copula functions, which include all of the parametric copulas suggested by Cherubini et al. (2004) and which permit a wide range of possible dependence structures between two prices of financial assets. To the best of our knowledge, firstly we consider almost all the single-parameter copulas investigated in prior financial applications and secondly each prior application considers at most three of our five functions.

Each of the copula functions has a single ${ }^{1}$ parameter, which determines the magnitude of the dependence. We make empirical comparisons between the Clayton, Frank, Gaussian, Gumbel, and Plackett copulas. All of these copulas can display either positive or negative dependence. The contours of the bivariate densities for $Y$ and $Z$ are shown on the left of Figure 1, when Spearman's $\rho$ equals 0.5 and the marginal distributions are standard normal; then $h(y, z)=c(\Phi(y), \Phi(z)) \phi(y) \phi(z)$ with $\phi(\cdot)$ and $\Phi(\cdot)$ representing the density and the c.d.f. of the standard normal distribution. The corresponding copula densities are shown on the right of Figure 1; the plotted variable is $c(\Phi(y), \Phi(z))$, which characterizes the dependence between $Y$ and $Z$.

The dependence structure is symmetric ${ }^{2}$ and tail-independent ${ }^{3}$ for the Frank, Gaussian, and Plackett copulas. Although the Gumbel copula has asymmetry and upper-tail dependence, the Clayton copula has asymmetry and

\footnotetext{
${ }^{1}$ One parameter is sufficient to model dependence. Multi-parameter copulas, such as the Student copula, are not investigated in this study because we rely on a single cross-rate option price when option information is used to infer the magnitude of the dependence between two exchange rates.

${ }^{2} \mathrm{~A}$ copula $C$ is symmetric if $(U, V)$ and $(1-U, 1-V)$ have the same c.d.f.

${ }^{3} \mathrm{~A}$ copula is tail-independent if $P(U>a \mid V>a)$ and $P(U<b \mid V<b)$ both converge to zero as $a \rightarrow 1$ and $b \rightarrow 0$.
} 

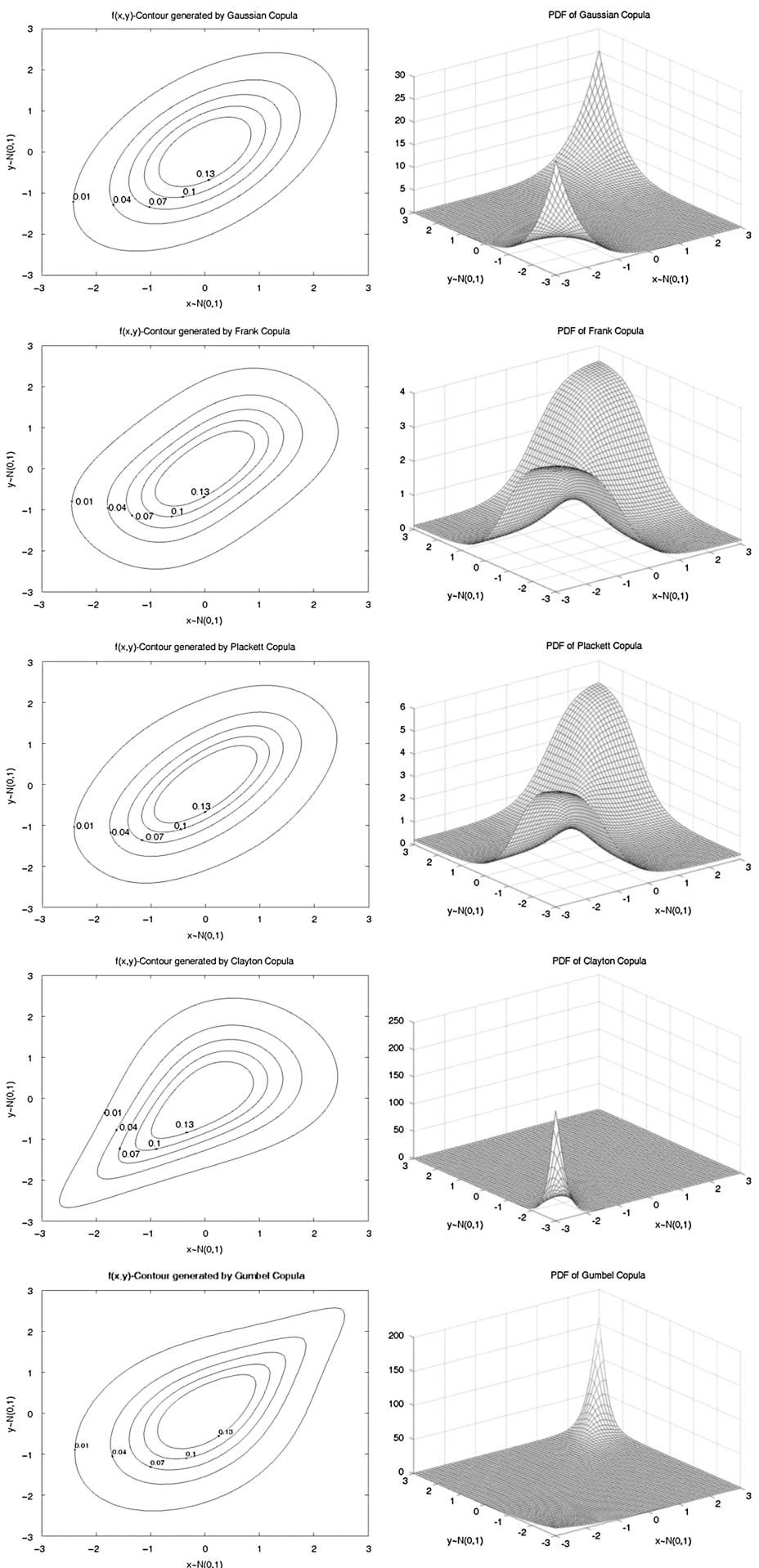

\section{FIGURE 1}

Bivariate copula densities. The contours are for the bivariate densities of two random variables whose marginal densities are the standard normal density. Each bivariate density is the product of the two marginal densities and a copula density function. Each copula density function is shown to the right of the corresponding contour plot for the bivariate density. Densities are shown for the Gaussian, Frank,

Plackett, Clayton, and Gumbel copulas when Spearman's $\rho$ equals 0.5. 
lower-tail dependence that has been used to model the dependence of equity prices (e.g. Cherubini and Luciano, 2002). Equations for all the copula functions are given in Appendix B.

\section{Estimation of the Dependence Parameter}

If the market price of one cross-rate option is available, typically the ATM option, then we can use numerical methods to obtain the implied estimate of the dependence parameter by equating the market price with the theoretical price given by (6). Otherwise, we can use a historical record of intraday dollardenominated currency returns for two exchange rates to calculate their realized correlation, using the method of Andersen, Bollerslev, Diebold, and Labys (2001). By assuming that the historical correlation equals the risk-neutral correlation in the future, we can estimate Spearman's $\rho$ for the risk-neutral Gaussian copula (see Appendix B). The dependence parameters for the other four copulas can then be obtained by constraining these copulas to have the same value for Spearman's $\rho$. We discuss the empirical relationship between the historical and risk-neutral correlations in the penultimate section.

When using intraday returns to estimate realized volatility or correlations, the choice of frequency is a tradeoff between selecting a high frequency and avoiding market microstructure effects. For our data, we find that the first-lag autocorrelations of the five-minute returns are significantly negative, which probably reflects microstructure effects. Consequently, we use 30-minute returns instead to calculate realized correlations as their first-lag autocorrelations are much lower. Following Andersen et al., the realized correlation coefficient between two sets of 30-minute returns, $\left\{r_{1, j}\right\}$ and $\left\{r_{2, j}\right\}$, whose latest return is at the end of day $t$, is given by the following formulae:

$$
\rho_{t}=\frac{\operatorname{Cov}_{t}}{\sigma_{1, t} \sigma_{2, t}}, \operatorname{Cov}_{t}=\sum_{j=1}^{n m} r_{1, j} r_{2, j} \quad \text { and } \quad \sigma_{i, t}^{2}=\sum_{j=1}^{n m} r_{i, j}^{2}
$$

where $n$ is the number of 30-minute intervals in one day and $m$ is the number of days used for estimation.

\section{METHODOLOGY FOR THE MARGINAL RNDS}

Marginal RNDs for the dollar prices of one pound and one euro are required when we construct their bivariate density using (11) and an appropriate copula density. Many specifications of univariate RNDs have been proposed, including lognormal mixtures (Liu, Shackleton, Taylor, \& Xu, 2007; Melick \& Thomas, 1997; Ritchey, 1990), generalized beta densities (Anagnou-Basioudis, Bedendo, 
Hodges, \& Tompkins, 2005; Liu et al., 2007), lognormal-polynomial densities (Madan \& Milne, 1994), multi-parameter discrete distributions (Jackwerth \& Rubinstein, 1996), and densities derived from fitting spline functions to implied volatilities (Bliss \& Panigirtzoglou, 2002). Provided options are traded for a range of exercise prices that encompass almost all of the risk-neutral distribution, several flexible density families will provide similar empirical estimates.

In our empirical discussion we focus on the results when each RND is a generalized beta density of the second kind (GB2). The GB2 density has only four parameters and many desirable properties: general levels of skewness and kurtosis are allowed, the shapes of the tails are fat relative to the lognormal density, and there are analytic formulae for the density, its moments, and the prices of options. Furthermore, parameter estimation is easy and does not involve any subjective choices, the estimated densities are never negative, and RNDs can be transformed analytically into real-world densities. Equations for RND and real-world densities, moments, and call prices are provided by Liu et al. (2007). The results for two other widely used RNDs, namely lognormal mixtures and lognormal-polynomial products, are also discussed later. Definitions, pricing formulae, and estimation methods for these three densities are given in Taylor (2005) and in Appendix C.

\section{EMPIRICAL FRAMEWORK}

Three steps are followed to obtain empirical estimates of the cross-rate RND and then the prices of cross-rate options. In the first step, we use market option prices for two dollar-denominated exchange rates, namely the dollar prices of one pound and one euro, to estimate their univariate RNDs with respect to the risk-neutral measure for the dollar numeraire. The two univariate RNDs and a one-parameter copula function are employed in the second step to obtain their bivariate RND, again for the dollar numeraire. The third step produces the cross-rate RND for the euro price of one pound, with respect to the risk-neutral measure for the euro numeraire. It also provides the prices of cross-rate options.

The cross-rate RND depends both on the choice of the copula function and the value of its dependence parameter. The parameter is estimated either from historical data or by matching the theoretical price of the ATM cross-rate option with its market price. The latter approach requires a numerical method that repeats the second and third steps until the parameter value is estimated accurately.

To assess our cross-rate RND and option pricing methodology, we consider one-month and three-month maturities. In addition, we also investigate the following two questions: 
(1) Which copula function(s) can model the dependence structure of exchange rates most satisfactorily?

(2) Is the information used to price cross-rate options efficiently shared across different markets for options on dollar exchange rates?

The cross-rate option prices generated by our copula formulae are compared with those for OTC cross-rate option prices. The differences between two sets of prices for the same options are summarized by three statistics: the Kolmogorov-Smirnov (K-S) statistic, which equals the maximum difference between two cumulative risk-neutral distribution functions, the average of the absolute differences between call prices as a ratio of the OTC prices (G-call), and the average magnitude of the difference between implied volatilities (G-implied).

\section{DATA}

The primary data are option prices for the $\$ / £, \$ / €$, and $€ / £$ exchange rates. It is not possible to obtain useful exchange-traded option prices for the $€ / £$ cross rate. Some cross-rate settlement prices are available for the CME, but they correspond to almost no trading volume. Consequently, we have to rely on OTC option prices for the cross rate. Such prices are not in the public domain to the best of our knowledge. We make use of a confidential file of OTC option price mid-quotes, supplied by the trading desk of an investment bank, which covers the period from May to December 2000. The OTC quotes are for all three exchange rates, recorded at the end of the day in London. There are typically prices for seven exercise prices, based upon "deltas" equal to $0.1,0.25,0.37$, $0.5,0.63,0.75$, and 0.9 .

A second source of $\$ / £$ and $\$ / €$ option prices is provided by CME settlement prices for options on currency futures contracts. These options are American. Their early exercise premia are estimated from the pricing approximation of Barone-Adesi and Whaley (1987), which allows us to deduce appropriate prices for European options. The average number of exercise prices for which there are option prices is 21 for the $\$ / £$ rate and 24 for the $\$ / €$ rate.

Our primary and secondary sources provide option prices for several exercise prices. We also study ATM prices from a third source for a longer period, until December 2007, but our supplier (namely Olsen and Associates) does not provide a useful number of out-of-the-money prices.

Figure 2 shows $\$ / €$ and $€ / £$ exchange rates from May to December 2000. It also displays the three-month, ATM implieds for these rates during this period. The averages of the ATM implieds are 9.8, 13.4, and 11.0\%, respectively, for the $\$ / £, \$ / €$, and $€ / £$ rates during the eight-month period. Implied volatility 

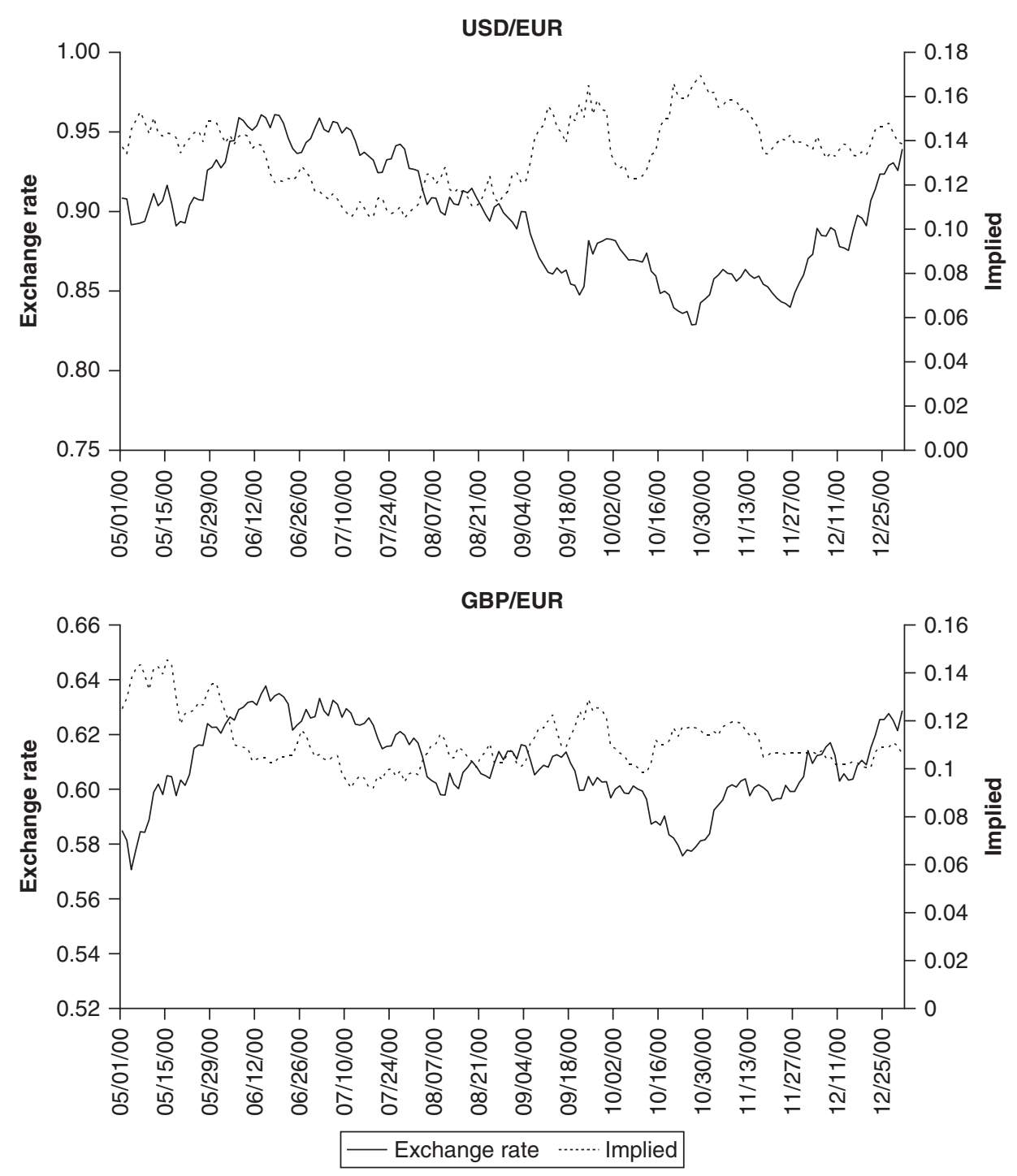

FIGURE 2

Time series of exchange rates and ATM implied volatilities for dollar/euro and pound/euro rates. Dollar/euro and pound/euro exchange rates and the three-month, ATM implied volatilities for these rates are shown from May to December 2000. ATM, at-the-money.

was lower, on average, during the subsequent seven-year period from 2001 to 2007; the corresponding averages for the longer period are 8.1, 9.4, and 6.4\%.

We generate both one-month and three-month RNDs. We obtain densities from the one-month OTC option prices for the first and third Tuesday of every month from May to October 2000. Thus our one-month results are based upon 
12 cases. Because options traded at the CME have fixed expiry dates, on the Fridays preceding the third Wednesdays of the contract months, it is impossible to match them with OTC options whose expiry dates are two business days before the forward dates. Therefore, we define matching CME option prices from the prices of the two nearest-to-expiry contracts that are more than one week from expiry. These matching prices are obtained by assuming that the implied volatilities have a linear term structure. ${ }^{4}$ There is less usable OTC data for the generation of the three-month densities. We use the three-month option prices on 12 June, 12 September, and 13 December 2000, from both the OTC source and the CME.

The OTC data and our processing of the CME data provide us with implied volatilities. The required one- and three-month forward rates, when the CME closes a few hours after the OTC quotes are recorded, are provided by the standard no-arbitrage equation that relates forward rates to spot prices and domestic and foreign interest rates. These forward rates are the expectations of the RNDs.

We also use the prices of $\$ / £$ and $\$ / €$ recorded every 30 minutes in the OTC markets to calculate one-month and three-month realized correlation coefficients for their returns from (12). Daily spot exchange rates and risk-free euro currency interest rates are obtained from Datastream.

\section{EMPIRICAL RESULTS}

Our empirical implementation uses two types of information to estimate the dependence parameter of the copula function. First we use the prices of ATM cross-rate options, then we assess the alternative strategy of relying on historical data to provide correlation estimates. Initially, we assume that all the marginal RNDs have GB2 distributions and subsequently we compare the GB2 results with those for other marginal RNDs. We also discuss a practical method for selecting the copula function. Finally, we consider possible explanations for a correlation risk premium revealed by our calculations.

\section{When the Prices of ATM Cross-Rate Options Are Used}

The properties of $€ / £$ cross-rate RNDs derived from OTC cross-rate option prices are shown in the first column of Table I and also in the first column of

\footnotetext{
${ }^{4}$ The term structure of the implied volatility of FX options is not flat and its slope changes frequently (Xu \& Taylor, 1994). For a selected exercise price, we approximate the CME implied volatility for an option that expires at time $T$ by $v=v_{1}+\left(v_{2}-v_{1}\right)\left(T-T_{1}\right) /\left(T_{2}-T_{1}\right)$ where $v_{1}$ and $v_{2}$ are the implied volatilities of the two near-to-expiry contracts, which have times $T_{1}$ and $T_{2}$ until expiry.
} 


\section{TABLE I}

Summary Statistics for Euro/Pound RNDs Obtained from Over-the-Counter Option Prices, Including One Cross-Rate Option Price

\begin{tabular}{|c|c|c|c|c|c|c|}
\hline Copula & Vanilla & Gaussian & Frank & Plackett & Clayton & Gumbel \\
\hline \multicolumn{7}{|c|}{ Panel A: Averages for one-month RNDs } \\
\hline$N$ & 12 & 12 & 12 & 12 & 12 & 12 \\
\hline$\rho / \theta$ & & 0.5609 & 4.2876 & 6.5994 & 1.2764 & 1.5721 \\
\hline Mean & 1.6399 & 1.6399 & 1.6399 & 1.6399 & 1.6399 & 1.6399 \\
\hline Variance & 0.0030 & 0.0029 & 0.0031 & 0.0032 & 0.0030 & 0.0030 \\
\hline Skewness & 0.2295 & 0.1089 & 0.1597 & 0.1824 & -0.1975 & 0.3467 \\
\hline Kurtosis & 4.2845 & 3.7081 & 4.9407 & 5.2758 & 4.5067 & 4.2712 \\
\hline$K-S^{a}$ & & 0.0140 & 0.0156 & 0.0213 & 0.0292 & 0.0119 \\
\hline G-call ${ }^{b}$ & & 0.0436 & 0.0325 & 0.0411 & 0.0645 & 0.0336 \\
\hline G-implied ${ }^{c}$ & & 0.0028 & 0.0026 & 0.0033 & 0.0053 & 0.0024 \\
\hline \multicolumn{7}{|c|}{ Panel B: Averages for three-month RNDs } \\
\hline$N$ & 3 & 3 & 3 & 3 & 3 & 3 \\
\hline$\rho / \theta$ & & 0.6580 & 5.4663 & 9.2415 & 1.7650 & 1.7570 \\
\hline Mean & 1.6207 & 1.6207 & 1.6207 & 1.6207 & 1.6207 & 1.6207 \\
\hline Variance & 0.0083 & 0.0079 & 0.0088 & 0.0092 & 0.0085 & 0.0083 \\
\hline Skewness & 0.2222 & 0.2042 & 0.2990 & 0.3438 & -0.1842 & 0.5369 \\
\hline Kurtosis & 4.3982 & 3.8037 & 5.4548 & 5.9379 & 4.8147 & 4.7460 \\
\hline$K-S^{a}$ & & 0.0120 & 0.0142 & 0.0219 & 0.0326 & 0.0197 \\
\hline G-call ${ }^{b}$ & & 0.0316 & 0.0356 & 0.0615 & 0.0625 & 0.0433 \\
\hline G-implied ${ }^{c}$ & & 0.0024 & 0.0022 & 0.0036 & 0.0054 & 0.0036 \\
\hline
\end{tabular}

Note. Average values of summary statistics for euro/pound risk-neutral densities, for various dependence functions. GB2 densities define the marginal dollar-rate densities and the vanilla cross-rate densities, all obtained from OTC option prices. The Gaussian, Frank, Plackett, Clayton, and Gumbel copula functions are used when defining the bivariate dollar-rate densities. The dependence parameters of the copulas are estimated by equating the theoretical at-the-money cross-rate option price with the market price. GB2, generalized beta density of the second kind; OTC, over-the-counter.

${ }^{a} \mathrm{~K}-\mathrm{S}$ : Kolmogorov-Smirnov statistic.

${ }^{b} G$-call: Average absolute error of call price as a ratio of the vanilla price.

${ }^{\mathrm{C}} \mathrm{G}$-implied: Average absolute error of implied volatility.

Table II. We refer to these as "vanilla" densities as they merely depend upon cross-rate market prices. Each vanilla density is derived from seven option prices. The remaining columns of Table I provide summary statistics for further cross-rate RNDs, each of which is obtained from seven OTC option prices for both the $\$ / £$ and the $\$ / €$ rates, a copula function, and the OTC price of the ATM $€ / £$ option. ${ }^{5}$ Likewise, the remaining columns of Table II summarize cross-rate RNDs obtained from CME option prices for the $\$ / £$ and the $\$ / €$ rates, a copula function, and the OTC price of the ATM $€ / £$ option.

The summary statistics are averages across densities. For example, each "Variance" statistic for a set of one-month densities is the average value of 12 variances, 1 for each RND. The statistics in Table I are generally similar to those in Table II.

${ }^{5} \mathrm{ATM}$ refers to options whose deltas equal 0.5. 
TABLE II

Summary Statistics for Euro/Pound RNDs Obtained from CME Option Prices and One Cross-Rate OTC Option Price

\begin{tabular}{|c|c|c|c|c|c|c|}
\hline Copula & Vanilla & Gaussian & Frank & Plackett & Clayton & Gumbel \\
\hline \multicolumn{7}{|c|}{ Panel A: Averages for one-month RNDs } \\
\hline$N$ & 12 & 12 & 12 & 12 & 12 & 12 \\
\hline$\rho / \theta$ & & 0.5699 & 4.3911 & 6.8340 & 1.2607 & 1.6990 \\
\hline Mean & 1.6399 & 1.6399 & 1.6399 & 1.6399 & 1.6399 & 1.6399 \\
\hline Variance & 0.0030 & 0.0029 & 0.0032 & 0.0032 & 0.0030 & 0.0030 \\
\hline Skewness & 0.2295 & 0.0512 & 0.0843 & 0.1024 & -0.3470 & 0.3416 \\
\hline Kurtosis & 4.2845 & 3.8997 & 5.1075 & 5.4711 & 4.6034 & 4.6467 \\
\hline $\mathrm{K}-\mathrm{S}^{\mathrm{a}}$ & & 0.0113 & 0.0198 & 0.0258 & 0.0378 & 0.0096 \\
\hline G-call ${ }^{b}$ & & 0.0389 & 0.0241 & 0.0390 & 0.0805 & 0.0253 \\
\hline G-implied ${ }^{c}$ & & 0.0022 & 0.0029 & 0.0040 & 0.0068 & 0.0018 \\
\hline \multicolumn{7}{|c|}{ Panel B: Averages for three-month RNDs } \\
\hline$N$ & 3 & 3 & 3 & 3 & 3 & 3 \\
\hline$\rho / \theta$ & & 0.6529 & 5.3352 & 9.9484 & 1.6479 & 1.7738 \\
\hline Mean & 1.6207 & 1.6207 & 1.6207 & 1.6207 & 1.6207 & 1.6207 \\
\hline Variance & 0.0083 & 0.0079 & 0.0087 & 0.0090 & 0.0082 & 0.0083 \\
\hline Skewness & 0.2222 & 0.1978 & 0.2643 & 0.2943 & -0.2906 & 0.5414 \\
\hline Kurtosis & 4.3982 & 3.7345 & 5.2226 & 5.6558 & 4.2536 & 4.7209 \\
\hline K-S ${ }^{a}$ & & 0.0087 & 0.0091 & 0.0169 & 0.0340 & 0.0105 \\
\hline G-call ${ }^{b}$ & & 0.0326 & 0.0231 & 0.0484 & 0.0888 & 0.0460 \\
\hline G-implied ${ }^{c}$ & & 0.0023 & 0.0015 & 0.0031 & 0.0063 & 0.0038 \\
\hline
\end{tabular}

Note. Average values of summary statistics for euro/pound risk-neutral densities, for various dependence functions. GB2 densities define the marginal dollar-rate densities obtained from CME option prices. The vanilla cross-rate densities are obtained from OTC option prices. The Gaussian, Frank, Plackett, Clayton, and Gumbel copula functions are used when defining the bivariate dollar-rate densities. The dependence parameters of the copulas are estimated by equating the theoretical at-the-money cross-rate option price with the market price. GB2, generalized beta density of the second kind; CME, Chicago Mercantile Exchange; OTC, over-the-counter.

${ }^{a} \mathrm{~K}-\mathrm{S}$ : Kolmogorov-Smirnov statistic.

${ }^{b} G$-call: Average absolute error of call price as a ratio of the vanilla price.

${ }^{\circ} \mathrm{G}$-implied: Average absolute error of implied volatility.

The means of all sets of 12 one-month densities are identical, because all the densities are risk-neutral, and likewise for all sets of 3 three-month densities. The average variances of the RNDs created from the various copula functions are very similar to those of the vanilla densities. There is more positive skewness in the vanilla densities than in all the sets of copula densities except the Gumbel density. The average skewness of the RNDs is positive and relatively high for the Gumbel copula, but low for the Frank, Gaussian, and Plackett copulas, whereas the averages are negative for the Clayton copula. The kurtosis averages for all sets of densities exceed three and the averages are fairly similar for the vanilla densities and those derived using copula functions. Generally, the cross-rate densities on a particular date are similar. This can be seen in Figure 3, which shows all the one-month densities formed on 5 September 2000; the cross-rate RNDs generated by the Frank, Gaussian, and Gumbel 

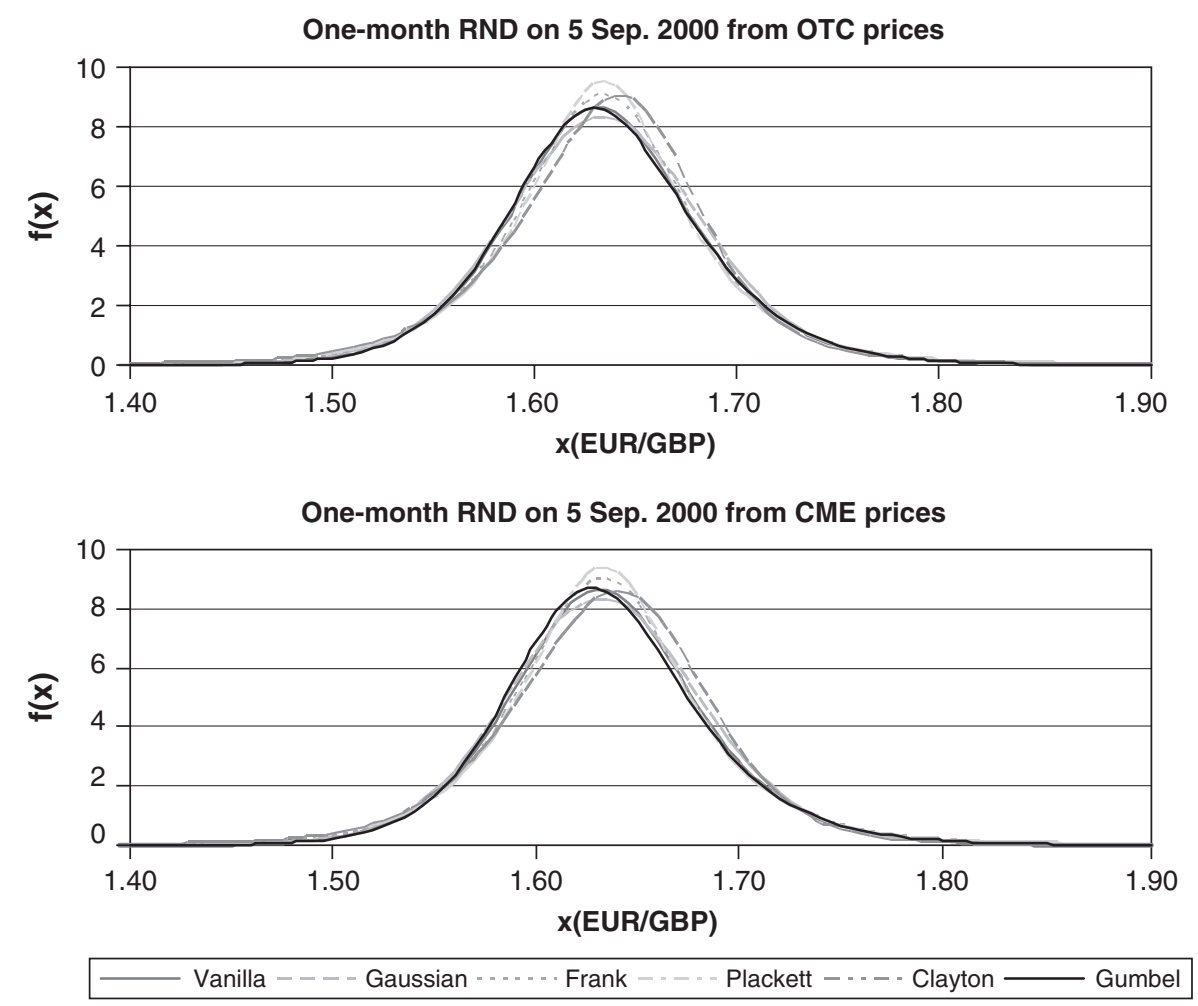

FIGURE 3

Euro/pound RNDs when one cross-rate option price is known. Typical RNDs for the euro/pound rate, respectively, derived from CME and OTC dollar-rate option prices. GB2 densities define the marginal dollar-rate densities and the vanilla cross-rate densities. The dependence parameters of the copulas are estimated by equating the theoretical at-the-money cross-rate option price with the market price. RND, risk-neutral density; CME, Chicago Mercantile Exchange; OTC, over-the-counter; GB2, generalized beta density of the second kind.

copulas are slightly nearer to the vanilla density than the densities obtained from the Clayton and Plackett copulas.

Next consider how near the densities derived from copulas are to the vanilla densities. The K-S statistic is an overall measure of the similarity of two densities. The averages of the K-S statistics are always least for the Frank, Gaussian, and Gumbel copulas and are then always less than 0.02. Similarity can also be assessed by comparing the option prices generated by different RNDs, for the options that are not ATM. For both the one-month and the three-month option contracts, the average absolute difference between a vanilla option price and a Frank option price is about 3\% of the vanilla option price, with slightly higher average differences for the Gaussian and Plackett option prices. The average differences for the Gumbel option prices are also about 3\% 
for the one-month contracts, but are higher for the three-month options. When implied volatilities are compared, the average absolute difference is less than the bid-ask spread (typically $0.5 \%$ ) for all but the Clayton copula. Our comparisons show that the Frank, Gaussian, and Gumbel copulas are satisfactory, with average absolute differences as low as $0.2 \%$; the Plackett copula is fairly satisfactory but the Clayton copula systematically performs less well.

Figure 4 shows averages of the implied volatility functions for the $€ / £$ cross rate, across density formation dates, for both OTC and CME dollar-rate data. These functions are plotted against the OTC option delta. All the copula functions generate volatility smiles. The vanilla densities and the Frank, Gaussian, and Plackett copulas all produce symmetric smiles. The Gumbel smiles are slightly asymmetric, but still close to the vanilla smiles, especially for the onemonth contracts. However, the Clayton smiles are highly asymmetric and hence are unsatisfactory. The curvature (or depth) of the smiles is highest for the Plackett smiles, and the Frank smiles are deeper than the Gaussian smiles. Generally, the market volatility smile, defined by the vanilla densities, lies above the Gaussian smile and below the Frank smile.

Figure 5 compares the average OTC and CME volatility smiles generated by the Frank and Gaussian copulas. The differences between the OTC and the CME average smiles are seen to be small, particularly when delta is between 25 and $75 \%$. The differences are all less than the bid-ask spread, even for the extreme values of delta. We conclude that the cross-rate densities obtained from the OTC and the CME option prices of dollar exchange rates are similar so that price information is efficiently shared by these two markets. Figure 5 also emphasizes that the Frank smiles are approximately twice as deep as the Gaussian smiles.

Our results show that the Frank and Gaussian copula functions are the most satisfactory when modeling the dependence between the risk-neutral distributions of the $\$ / £$ and the $\$ / €$ exchange rates for both the one-month and the threemonth densities. The Gumbel copula function also performs well for the onemonth densities. The deeper smiles produced by the Plackett copula show that it is less satisfactory. Because the implied volatility pattern of foreign exchange rates is a smile, rather than a smirk, the highly asymmetric Clayton copula appears to be unsatisfactory, although it is often used to model the dependence structure of equity prices satisfactorily (e.g. Cherubini \& Luciano, 2002).

\section{When the Prices of ATM Cross-Rate Options Are Not Used}

The previous strategy of estimating the dependence parameter by matching the theoretical ATM call price with the market price is like using an implied 

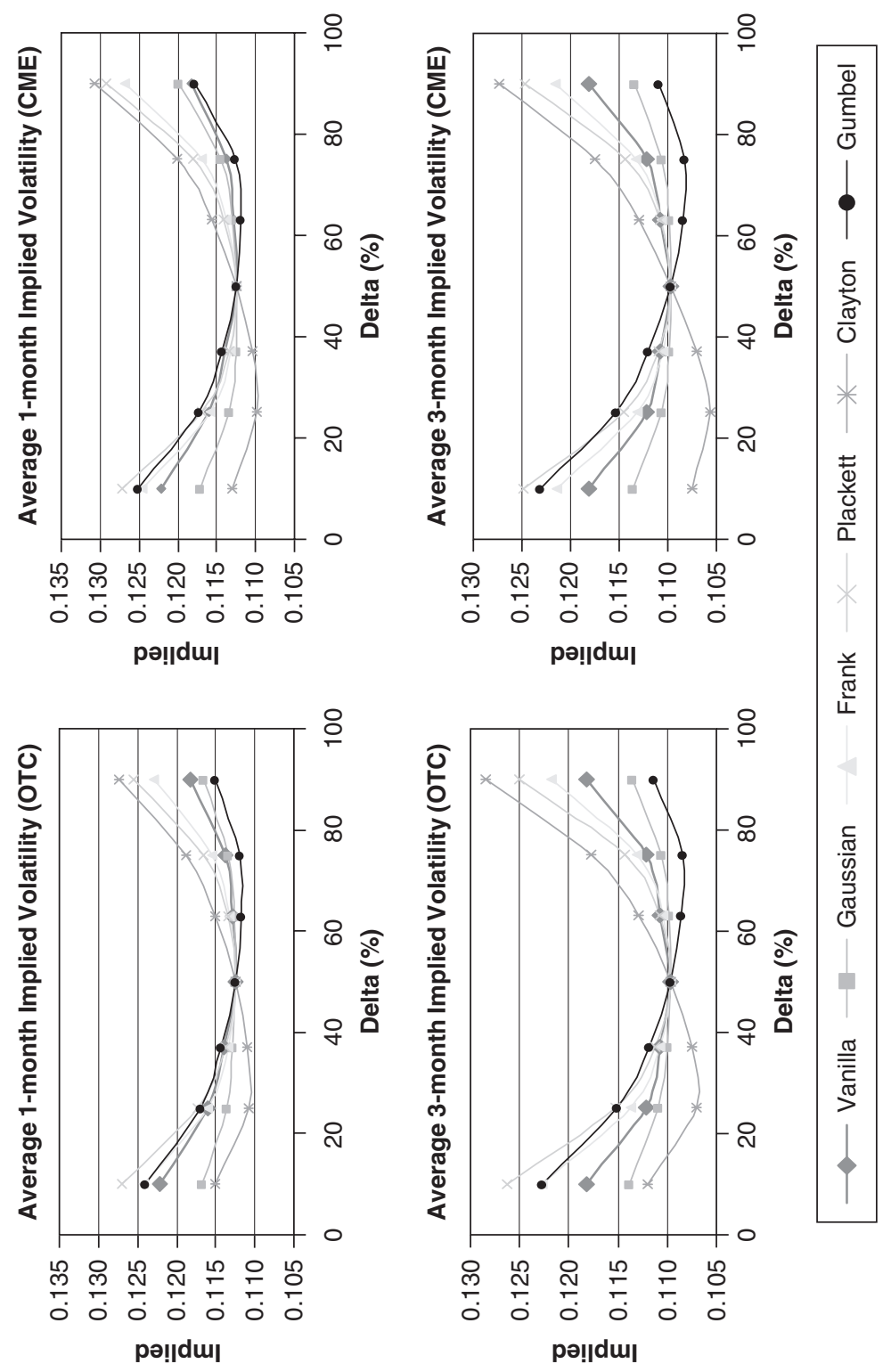

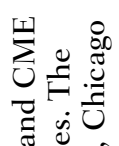

0

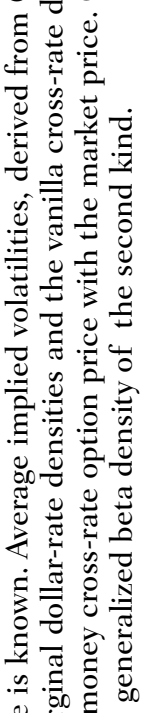

岑.

릉

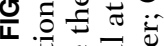

政

$\triangle \cong$

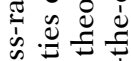

की

政

ปั

원 ⿹ㅝㅁ잉

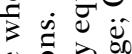

을 긍

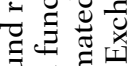

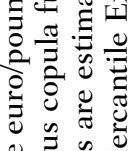

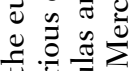

कृ

电

过苛

흥

政

产它节 

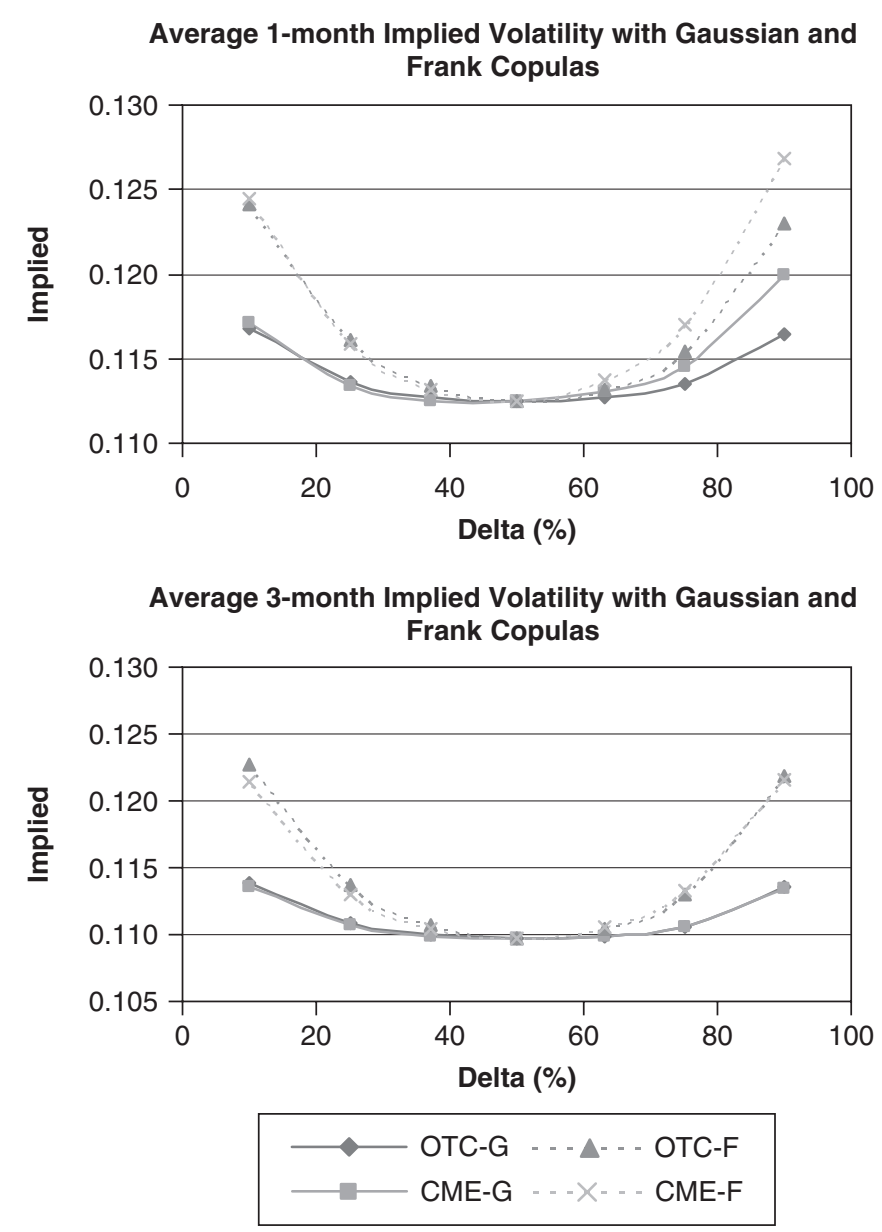

FIGURE 5

Implied volatilities for the euro/pound rate from OTC and CME option prices, when one cross-rate option price is known. Average implied volatilities, derived from OTC and CME option prices and various copula functions. GB2 densities define the marginal dollar-rate densities and the vanilla crossrate densities. The parameters of the copulas are estimated by equating the theoretical at-the-money cross-rate option price with the market price. CME, Chicago Mercantile Exchange; OTC, over-thecounter; GB2, generalized beta density of the second kind.

risk-neutral correlation to estimate the dependence parameter. When the ATM cross-rate market price is either unavailable or considered to be an unfair price, we can instead use the realized historical correlation to estimate the dependence parameter.

Time series of implied and realized correlation coefficients through our eight-month sample period in 2000 are shown in Figure 6. These are correlations between changes in the logarithms of the $\$ / £$ and the $\$ / €$ rates. 


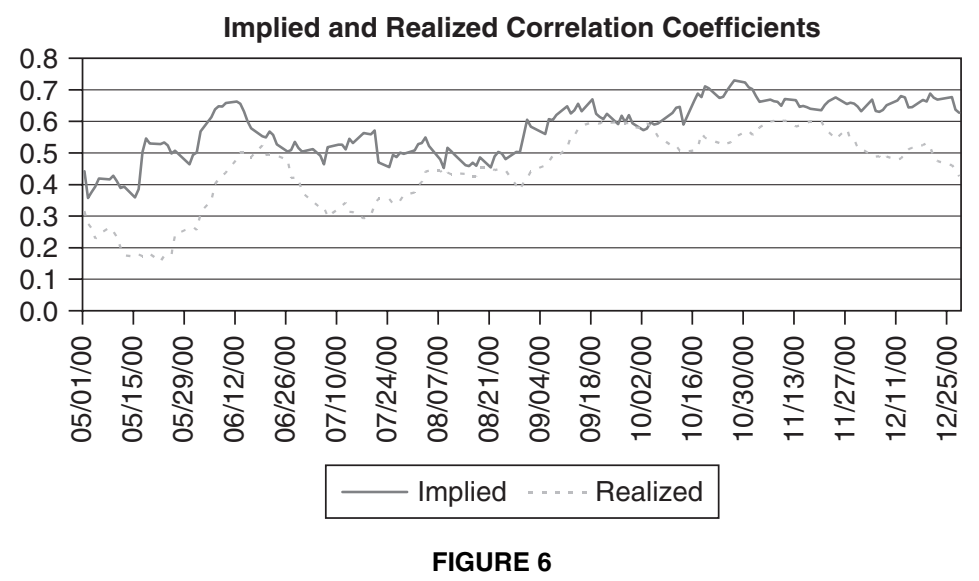

Time series of implied and realized correlation coefficients for the dollar/pound and dollar/euro rates. Each implied correlation is calculated from the one-month at-the-money implied volatilities of OTC options on the dollar/pound, dollar/euro, and euro/pound rates. Each realized correlation is calculated from the previous month of 30-minute returns, obtained from CME dollar/pound and dollar/euro futures prices. CME, Chicago Mercantile Exchange; OTC, over-the-counter.

The implied correlations are derived directly from the ATM implied volatilities of $\$ / £, \$ / €$, and $€ / £$ OTC options one month from expiry, whereas the realized correlations are calculated from the previous month of 30-minute ${ }^{6}$ returns for the $\$ / £$ and the $\$ / €$ rates.

It is seen very clearly that the realized correlations are lower than the implied correlations; we defer discussion of why this occurs until the end of this empirical section. The immediate consequence of the differences between the implied and realized correlations is that the differences between the vanilla and the copula results will increase, for densities, option prices, and implied volatilities.

The properties of $€ / £$ cross-rate RNDs derived from option prices for the $\$ / £$ and the $\$ / €$ rates, a copula function, and the historical correlation are summarized in Tables III and IV, respectively, for densities derived from OTC and CME prices. These summary statistics can be compared with those presented in Tables I and II. The densities are guaranteed to remain risk-neutral. The most obvious difference, which occurs when historical correlations are used, is that the variances of the cross-rate RNDs increase significantly due to the lower dependence between the marginal dollar-rate densities. It is now hard to tell which copula is the most satisfactory, regardless of the criterion, because all the copula functions produce option prices that are evidently different from the

\footnotetext{
${ }^{6}$ The realized correlations are almost unchanged if the frequency of returns is switched from 30 to 60 minutes. The average realized correlations are 0.45 and 0.49 for these two frequencies and the period covered by Figure 6.
} 
TABLE III

Summary Statistics for Euro/Pound RNDs Obtained from Over-the-Counter Option Prices and the Historical Realized Correlation Coefficient

\begin{tabular}{|c|c|c|c|c|c|}
\hline Copula & Gaussian & Frank & Plackett & Clayton & Gumbel \\
\hline \multicolumn{6}{|c|}{ Panel A: Averages for one-month RNDs } \\
\hline$N$ & 12 & 12 & 12 & 12 & 12 \\
\hline$\rho / \theta$ & 0.4688 & 3.1475 & 4.9331 & 0.9855 & 1.4950 \\
\hline Mean & 1.6399 & 1.6399 & 1.6399 & 1.6399 & 1.6399 \\
\hline Variance & 0.0034 & 0.0037 & 0.0037 & 0.0034 & 0.0032 \\
\hline Skewness & 0.1213 & 0.1646 & 0.1793 & -0.1152 & 0.3246 \\
\hline Kurtosis & 3.6955 & 4.5734 & 4.8866 & 4.2726 & 4.2081 \\
\hline $\mathrm{K}-\mathrm{S}^{\mathrm{a}}$ & 0.0287 & 0.0247 & 0.0241 & 0.0349 & 0.0193 \\
\hline G-call ${ }^{b}$ & 0.1080 & 0.1503 & 0.1365 & 0.1036 & 0.0917 \\
\hline G-implied ${ }^{c}$ & 0.0090 & 0.0125 & 0.0111 & 0.0101 & 0.0069 \\
\hline \multicolumn{6}{|c|}{ Panel B: Averages for three-month RNDs } \\
\hline$N$ & 3 & 3 & 3 & 3 & 3 \\
\hline$\rho / \theta$ & 0.4845 & 3.2342 & 4.9341 & 1.0087 & 1.5069 \\
\hline Mean & 1.6207 & 1.6207 & 1.6207 & 1.6207 & 1.6207 \\
\hline Variance & 0.0114 & 0.0125 & 0.0124 & 0.0116 & 0.0108 \\
\hline Skewness & 0.2478 & 0.3183 & 0.3399 & 0.0202 & 0.4686 \\
\hline Kurtosis & 3.7751 & 4.7007 & 5.0252 & 4.2224 & 4.4455 \\
\hline $\mathrm{K}-\mathrm{S}^{\mathrm{a}}$ & 0.0486 & 0.0448 & 0.0395 & 0.0460 & 0.0411 \\
\hline G-callb & 0.2535 & 0.3226 & 0.3055 & 0.2309 & 0.2295 \\
\hline G-implied ${ }^{c}$ & 0.0195 & 0.0239 & 0.0223 & 0.0201 & 0.0159 \\
\hline
\end{tabular}

Note. Average values of summary statistics for euro/pound risk-neutral densities, for various dependence functions. GB2 densities define the marginal dollar-rate densities and the vanilla cross-rate densities, all obtained from OTC option prices. The Gaussian, Frank, Plackett, Clayton, and Gumbel copula functions are used when defining the bivariate dollar-rate densities. The dependence parameters of the copulas for one-month (threemonth) maturities are estimated from the historical correlation for one month (three months) of 30-minute dollarrate returns. GB2, generalized beta density of the second kind; OTC, over-the-counter.

${ }^{a} \mathrm{~K}-\mathrm{S}$ : Kolmogorov-Smirnov statistics.

${ }^{b} G$-call: Average absolute error of call price as a ratio of the vanilla price.

${ }^{\mathrm{C}} \mathrm{G}$-implied: Average absolute error of implied volatility.

vanilla option prices. For example, the average absolute difference between the copula and vanilla implied volatilities is about $1 \%$ for the one-month maturities and $2 \%$ for the three-month maturities when historical correlations are employed.

One-month cross-rate RNDs formed on a typical date, 18 July 2000, are shown in Figure 7. The peaks of the copula densities are lower than the vanilla densities because the copula variances exceed the vanilla variances. Figure 8 shows the averages of the one-month implied volatility functions. All the copula functions again produce volatility smiles, although the general levels of these smiles are visibly higher than the vanilla smiles. The previous remarks about the relative depth and the symmetry of the copula smiles remain valid when the implied correlation is replaced by the historical correlation. 
TABLE IV

Summary Statistics for Euro/Pound RNDs Obtained from CME Option Prices and the Historical Realized Correlation Coefficient

\begin{tabular}{|c|c|c|c|c|c|}
\hline Copula & Gaussian & Frank & Plackett & Clayton & Gumbel \\
\hline \multicolumn{6}{|c|}{ Panel A: Averages for one-month RNDs } \\
\hline$N$ & 12 & 12 & 12 & 12 & 12 \\
\hline$\rho / \theta$ & 0.4688 & 3.1475 & 4.9331 & 0.9855 & 1.4950 \\
\hline Mean & 1.6399 & 1.6399 & 1.6399 & 1.6399 & 1.6399 \\
\hline Variance & 0.0035 & 0.0038 & 0.0038 & 0.0035 & 0.0034 \\
\hline Skewness & 0.0718 & 0.1042 & 0.1147 & -0.2385 & 0.3001 \\
\hline Kurtosis & 3.8751 & 4.7981 & 5.1415 & 4.3590 & 4.5417 \\
\hline $\mathrm{K}-\mathrm{S}^{\mathrm{a}}$ & 0.0257 & 0.0243 & 0.0244 & 0.0369 & 0.0186 \\
\hline G-call ${ }^{b}$ & 0.1052 & 0.1590 & 0.1456 & 0.1017 & 0.1045 \\
\hline G-implied ${ }^{\mathrm{C}}$ & 0.0100 & 0.0140 & 0.0127 & 0.0106 & 0.0086 \\
\hline \multicolumn{6}{|c|}{ Panel B: Averages for three-month RNDs } \\
\hline$N$ & 3 & 3 & 3 & 3 & 3 \\
\hline$\rho / \theta$ & 0.4845 & 3.2342 & 4.9341 & 1.0087 & 1.5069 \\
\hline Mean & 1.6207 & 1.6207 & 1.6207 & 1.6207 & 1.6208 \\
\hline Variance & 0.0111 & 0.0122 & 0.0120 & 0.0109 & 0.0109 \\
\hline Skewness & 0.2294 & 0.2796 & 0.2944 & -0.0765 & 0.4617 \\
\hline Kurtosis & 3.6950 & 4.5508 & 4.8499 & 3.8675 & 4.4056 \\
\hline $\mathrm{K}-\mathrm{S}^{\mathrm{a}}$ & 0.0464 & 0.0425 & 0.0375 & 0.0467 & 0.0413 \\
\hline G-call ${ }^{b}$ & 0.2313 & 0.2927 & 0.2771 & 0.1988 & 0.2319 \\
\hline G-implied $^{\mathrm{C}}$ & 0.0181 & 0.0222 & 0.0207 & 0.0176 & 0.0162 \\
\hline
\end{tabular}

Note. Average values of summary statistics for euro/pound risk-neutral densities, for various dependence functions. GB2 densities define the marginal dollar-rate densities obtained from CME option prices. The vanilla cross-rate densities are obtained from OTC option prices. The Gaussian, Frank, Plackett, Clayton, and Gumbel copula functions are used when defining the bivariate dollar-rate densities. The dependence parameters of the copulas for one-month (three-month) maturities are estimated from the historical correlation for one month (three months) of 30-minute dollar-rate returns. GB2, generalized beta density of the second kind; CME, Chicago Mercantile Exchange; OTC, over-the-counter.

${ }^{a} \mathrm{~K}-\mathrm{S}$ : Kolmogorov-Smirnov statistics.

${ }^{b} \mathrm{G}$-call: Average absolute error of call price as a ratio of the vanilla price.

${ }^{\circ} \mathrm{G}$-implied: Average absolute error of implied volatility.

It is difficult to decide which copula function performs best when historical data are used to estimate correlations. We do see that the average absolute differences between copula and vanilla implied volatilities are lowest for the Gaussian and Gumbel copulas with the latter fitting slightly better. The average absolute differences of call prices are also comparatively low for these two copulas, whereas the K-S statistics favor the Gumbel copula. We also see that the shapes of the vanilla volatility smiles are most similar to those of Frank, Gaussian, and Gumbel volatility smiles. Therefore, the Gaussian and Gumbel copulas should perform satisfactorily if the precision of historical correlation estimates can be improved. 

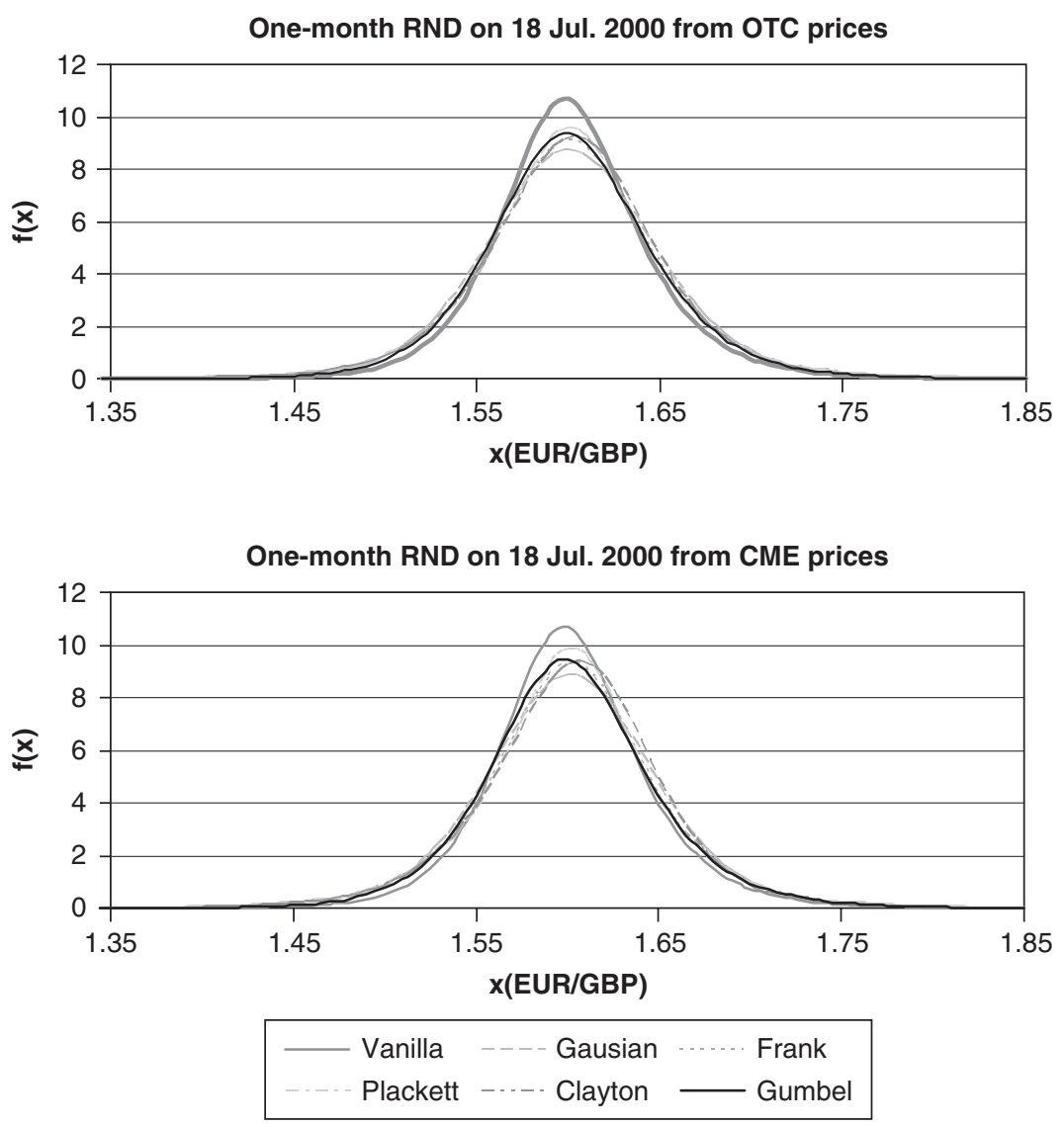

FIGURE 7

Euro/pound RNDs when dependence is estimated from historical prices. Typical RNDs for the euro/pound rate, respectively, derived from CME and OTC dollar-rate option prices. GB2 densities define the marginal dollar-rate densities and the vanilla cross-rate densities. The dependence parameters of the copulas are estimated from the historical correlation for one month of 30-minute dollar-rate returns. RND, risk-neutral density; CME, Chicago Mercantile Exchange; OTC, over-the-counter; GB2, generalized beta density of the second kind.

\section{Robustness Analysis}

Comparisons of different methods for estimating univariate RNDs (e.g. Campa et al., 1998) have shown that most methods produce similar estimates, provided options are traded for a range of strike prices that encompass almost all of the RND. To check if our cross-rate density and option results derived from a bivariate distribution depend on the GB2 assumption for the marginal RNDs, we compare our previous results for one-month OTC options when one crossrate option price is available with those obtained by assuming either lognormalmixture or lognormal-polynomial RNDs for the dollar rates. 

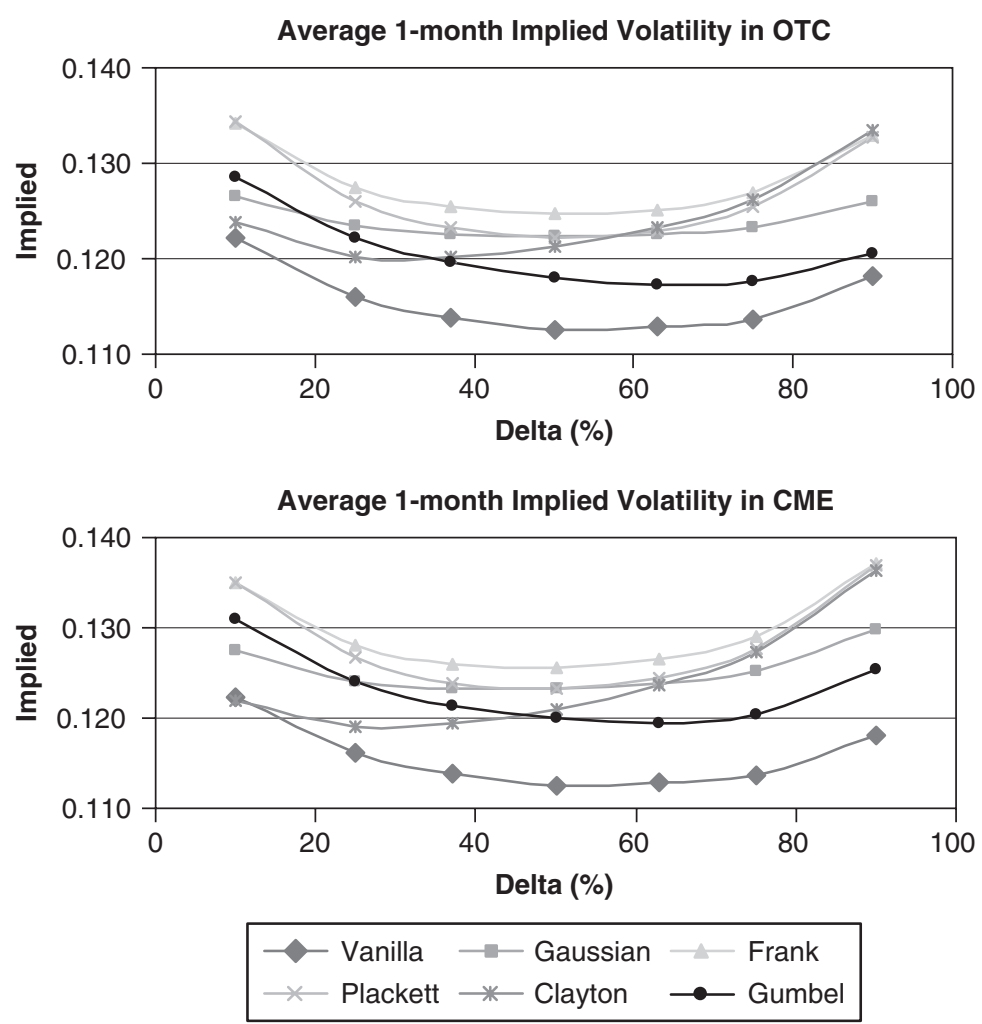

FIGURE 8

Implied volatilities for the euro/pound rate, when dependence is estimated from historical prices. Average one-month implied volatilities, derived from OTC and CME option prices and various copula functions. GB2 densities define the marginal dollar-rate densities and the vanilla cross-rate densities. The dependence parameters of the copulas are estimated from the historical correlation for one month of 30-minute dollar-rate returns. CME, Chicago Mercantile Exchange; OTC, over-the-counter; GB2, generalized beta density of the second kind.

The summary statistics for the cross-rate RNDs derived from the three distributional assumptions and the five copula functions are shown in Table V. There are no important differences between the cross-rate RND densities derived from the three families of marginal densities. Also, the averages of the K-S statistics, the absolute differences between call prices, and the magnitudes of the differences between implied volatilities are very similar across distributional assumptions. Furthermore, these numerical measures of agreement between densities are very small and negligible when we compare two sets of cross-rate densities obtained from the same copula but different marginal RNDs. For example, the largest K-S statistic is only 0.0025 and the largest difference of average implied volatilities is only $0.02 \%$, whereas Figure 9 illustrates the similarities of the cross-rate RNDs and the implied volatilities. 


\section{TABLE V}

Summary Statistics for Euro/Pound RNDs with Various Distributional Assumptions

\begin{tabular}{lccccc}
\hline Copula & Gaussian & Frank & Plackett & Clayton & Gumbel \\
\hline Panel A: GB2 & densities & & & & \\
$N$ & 12 & 12 & 12 & 12 & 12 \\
$\rho / \theta$ & 0.5609 & 4.2876 & 6.5994 & 1.2764 & 1.5721 \\
Mean & 1.6399 & 1.6399 & 1.6399 & 1.6399 & 1.6399 \\
Variance & 0.0029 & 0.0031 & 0.0032 & 0.0030 & 0.0030 \\
Skewness & 0.1089 & 0.1597 & 0.1824 & -0.1975 & 0.3467 \\
Kurtosis $^{\text {K-S }}$ & 3.7081 & 4.9407 & 5.2758 & 4.5067 & 4.2712 \\
G-call $^{\text {b }}$ & 0.0140 & 0.0156 & 0.0213 & 0.0292 & 0.0119 \\
G-implied $^{\text {c }}$ & 0.0436 & 0.0325 & 0.0411 & 0.0645 & 0.0336 \\
& 0.0028 & 0.0026 & 0.0033 & 0.0053 & 0.0024
\end{tabular}

Panel B: Lognormal-mixture densities

\begin{tabular}{lccccc}
$N$ & 12 & 12 & 12 & 12 & 12 \\
$\rho / \theta$ & 0.5592 & 4.2562 & 6.5222 & 1.2700 & 1.5715 \\
Mean & 1.6399 & 1.6399 & 1.6399 & 1.6399 & 1.6399 \\
Variance & 0.0028 & 0.0030 & 0.0031 & 0.0030 & 0.0030 \\
Skewness & 0.0903 & 0.1105 & 0.1296 & -0.1966 & 0.3345 \\
Kurtosis & 3.1101 & 3.9153 & 4.2272 & 3.2493 & 4.1858 \\
K-S $^{\mathrm{a}}$ & 0.0155 & 0.0149 & 0.0200 & 0.0304 & 0.0123 \\
G-call $^{b}$ & 0.0474 & 0.0331 & 0.0398 & 0.0712 & 0.0335 \\
G-implied $^{\mathrm{c}}$ & 0.0030 & 0.0025 & 0.0031 & 0.0055 & 0.0024 \\
Panel C: Lognormal-polynomial densities & & & \\
$N$ & 12 & 12 & 12 & 12 & 12 \\
$\rho / \theta$ & 0.5604 & 4.2781 & 6.5766 & 1.2744 & 1.5735 \\
Mean & 1.6399 & 1.6399 & 1.6399 & 1.6399 & 1.6399 \\
Variance & 0.0028 & 0.0031 & 0.0032 & 0.0030 & 0.0029 \\
Skewness & 0.1023 & 0.1375 & 0.1613 & -0.2088 & 0.3331 \\
Kurtosis & 3.5256 & 4.5273 & 4.8863 & 4.1847 & 4.1250 \\
K-S & 0.0130 & 0.0161 & 0.0218 & 0.0292 & 0.0118 \\
G-call $^{\mathrm{b}}$ & 0.0445 & 0.0324 & 0.0398 & 0.0671 & 0.0336 \\
G-implied $^{\mathrm{C}}$ & 0.0028 & 0.0026 & 0.0032 & 0.0054 & 0.0024 \\
\hline
\end{tabular}

Note. Average values of summary statistics for euro/pound risk-neutral densities, for various distributional assumptions and dependence functions. Either GB2, lognormal mixtures, or lognormal-polynomial densities define the marginal dollar-rate densities and the vanilla cross-rate densities, all obtained from one-month OTC option prices. The Gaussian, Frank, Plackett, Clayton, and Gumbel copula functions are used when defining the bivariate dollar-rate densities. The dependence parameters of the copulas are estimated by equating the theoretical at-the-money cross-rate option price with the market price. GB2, generalized beta density of the second kind; OTC, over-the-counter.

${ }^{a} \mathrm{~K}-\mathrm{S}$ : Kolmogorov-Smirnov statistic.

${ }^{b} G$-call: Average absolute error of call price as a ratio of the vanilla price.

${ }^{\circ} \mathrm{G}$-implied: Average absolute error of implied volatility.

Therefore, our results are not sensitive to the distributional assumption applied to the dollar-rate RNDs.

As it is difficult to obtain the prices of OTC cross-rate options, our empirical study only covers the $€ / £$ cross rate. Although our results imply that the Frank, Gaussian, and Gumbel copulas can model the dependence structure 


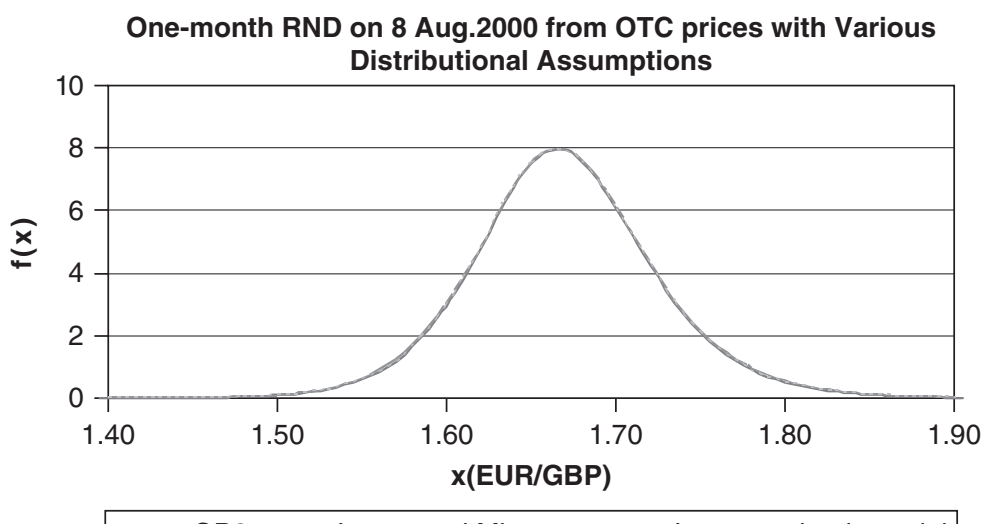

GB2 ---- Lognormal Mixtures …... Lognormal-polynomial

Average 1-month Implied Volatility with Various Distributional Assumptions

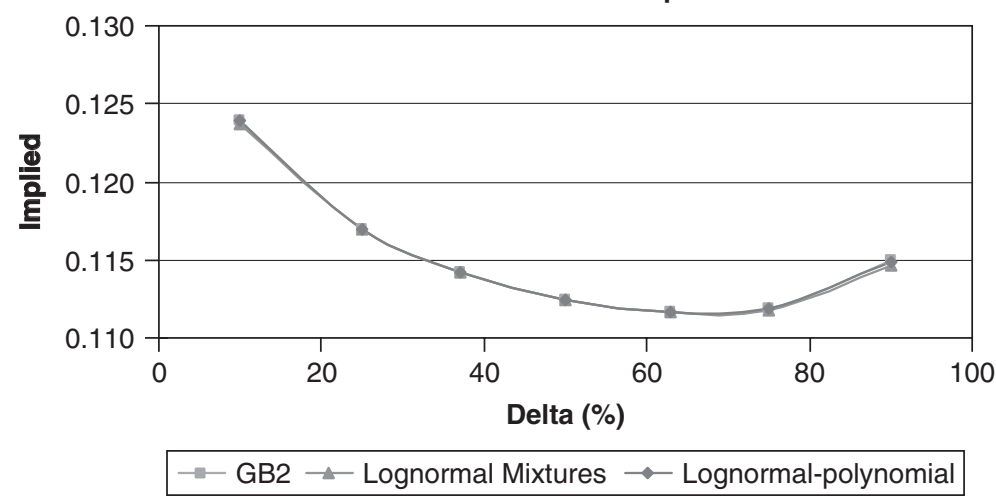

FIGURE 9

Euro/pound RNDs and implied volatilities from OTC option prices with various distributional assumptions, when one cross-rate option price is known. Typical one-month RNDs and average implied volatilities for the euro/pound rate derived from OTC dollar-rate option prices with various distribution assumptions and the Gumbel copula function. Either GB2, lognormal mixtures, or lognormal-polynomial densities define the marginal dollar-rate densities and the vanilla cross-rate densities. The parameters of the copulas are estimated by equating the theoretical at-the-money cross-rate option price with the market price. RND, risk-neutral density; OTC, over-the-counter; GB2, generalized beta density of the second kind.

of the $\$ / £$ and the $\$ / €$ rates satisfactorily, it is possible that different pairs of exchange rates are associated with different dependence structures. Therefore, identifying a way to select a suitable copula function for other pairs of exchange rates when option prices for their cross rates are not available is an important issue.

It may be reasonable to assume that the dependence structures of both the RND and real-world densities come from the same copula families, and thus it is plausible to infer the appropriate copula function from the historical prices of the two exchange rates. We have explored this strategy by estimating the 
GARCH-copula dependence model of Bartram et al. (2007). In our implementation, the returns from each exchange rate are modeled by supposing they follow an $\mathrm{MA}(1)$ process, with the conditional variance specified as $\operatorname{GARCH}(1,1)$ and the conditional density as Student- $t$. This model specifies univariate, conditional c.d.f. for each exchange rate from which time series of observed cumulative probabilities can be calculated, say $\left\{u_{t}\right\}$, for the $\$ / £$ rate and $\left\{v_{t}\right\}$ for the $\$ / €$ rate. A time-varying copula parameter, such as $\rho_{t}$ for the Gaussian copula, can then be estimated by an equation resembling an autoregressive process. Like Bartram et al. (2007), we suppose that

$$
\rho_{t}=\omega+\phi_{1} \rho_{t-1}+\phi_{2} \rho_{t-2}+\gamma\left|u_{t-1}-v_{t-1}\right| \text {. }
$$

The parameters in this equation are estimated by maximizing the loglikelihood of the bivariate time series $\left\{u_{t}, v_{t}\right\}$.

We find that the highest log-likelihood for the bivariate data set of $\$ / £$ and $\$ / €$ daily returns from 2000 to 2007 is provided by the Gaussian copula. The differences between the log-likelihood for the Gaussian copula and the loglikelihoods for the other copulas are 21 for the Gumbel copula, 32 for the Plackett copula, 97 for the Frank copula, and 217 for the Clayton copula; a similar ranking of the copulas was reported in a previous version of this article for the earlier period from 1994 to 2000. The likelihood results are consistent with the results obtained from the cross-rate vanilla option prices.

Figure 10 shows the time series of $\rho_{t}$ for the Gaussian copula, calculated using (13) and the maximum-likelihood parameter values, which are $\omega=0.0215$, $\phi_{1}=0.9806, \phi_{2}=0$, and $\gamma=-0.0428$.

\section{Determinants of the Correlation Risk Premium}

Figure 6 shows that the implied correlation between returns for the $\$ / £$ and the $\$ / €$ rates generally exceeded the realized correlation during 2000 . Figure 11 shows that this is also true for the longer and more recent period from 2001 to $2007 .^{7}$ The differences between the implied and realized correlations can be interpreted as estimates of the correlation risk premium and this premium can be important. ${ }^{8}$

Some possible explanations for these differences are biased historical estimates, mispriced ATM cross-rate options, and premia for risks that cannot be hedged. Firstly, historical estimates may contain bias that can arise from

\footnotetext{
${ }^{7}$ We thank the referee for advising us to consider the longer period and how to evaluate empirical explanations for the differences between implied and realized correlations.

${ }^{8}$ For example, suppose that the volatility of all dollar rates is $10 \%$ and that these rates do not have a volatility risk premium. Then implied and realized correlations equal to 0.6 and 0.5 , respectively, provide respective cross-rate volatilities equal to 8.9 and $10.0 \%$, i.e. they imply an economically significant and negative crossrate volatility risk premium.
} 


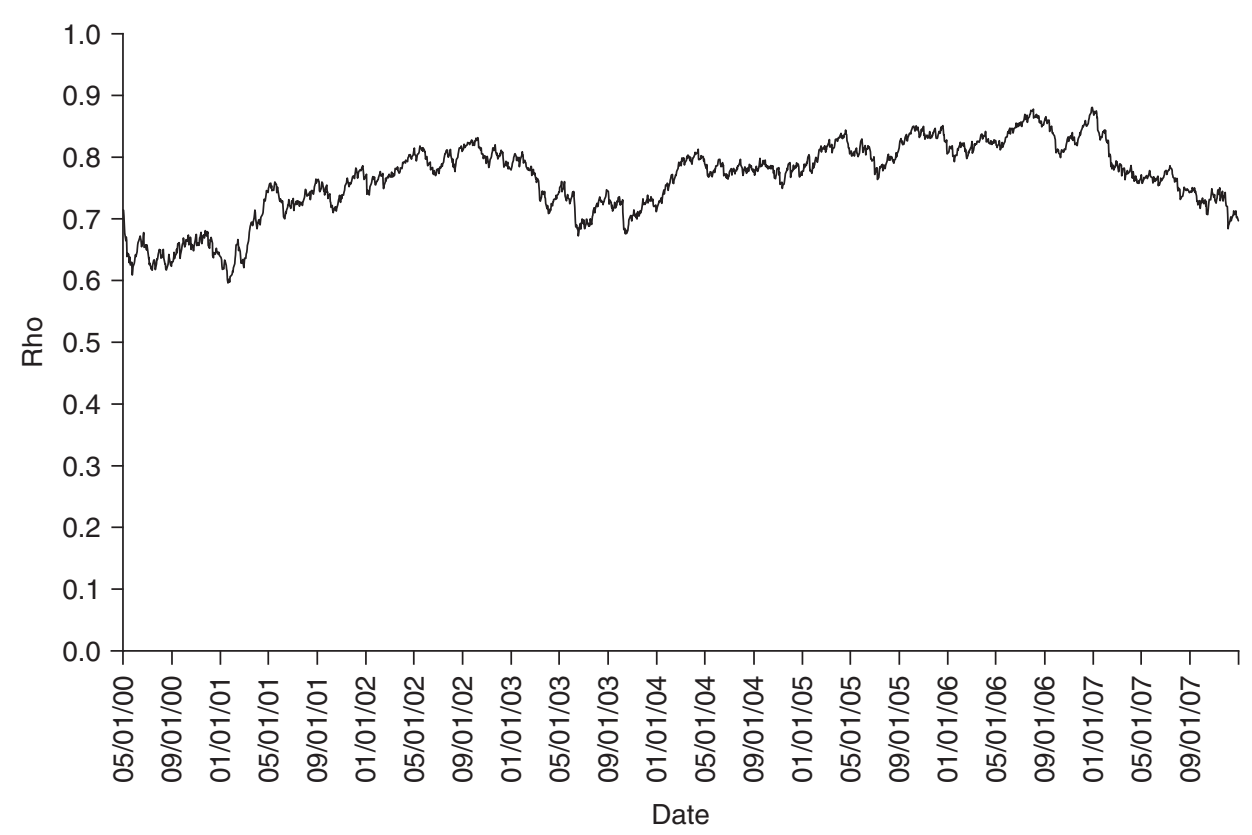

FIGURE 10

Time series of the dependence parameter for the Gaussian copula. The time series of the dependence parameter, $\rho_{t}$, for the Gaussian copula is produced for the dollar/pound and dollar/euro rates from 2000 to 2007.

bid-ask effects and non-synchronous returns. Secondly, the quotes of crossrate options may differ from their fundamental prices because of market liquidity and clientele effects. Thirdly, when asset prices follow simple diffusion processes, the second-moment measures of returns, such as variances and correlations, do not depend on risk assumptions. Thus, variances and correlations are then the same under the real-world measure $P$ and the risk-neutral measure Q. However, as suggested by the theoretical work of Branger and Schlag (2004), when price processes include jump components, the change of measure will affect variances and correlations and the size of this impact varies across maturities. Furthermore, if correlations follow a diffusion process then correlation risk can explain why expected correlations are different under measures $P$ and $Q$; see the equity market analysis of Driessen, Maenhout, and Vilkov (2009).

Let $d_{t}$ denote the correlation difference on day $t$, defined as the threemonth ATM implied correlation minus the realized correlation calculated from 30-minute returns. We attempt to explain the variation in $d_{t}$ by a regression model, which includes three-month interest rates (denoted by $i_{\$, t}, i_{€, t}, i_{€, t}$ ), the cross-rate implied volatility (denoted by $I_{t}$ ), the historical volatility proxied by the cross-rate absolute return (denoted by $\left|r_{t}\right|$ ), and a proxy for cross-rate price 


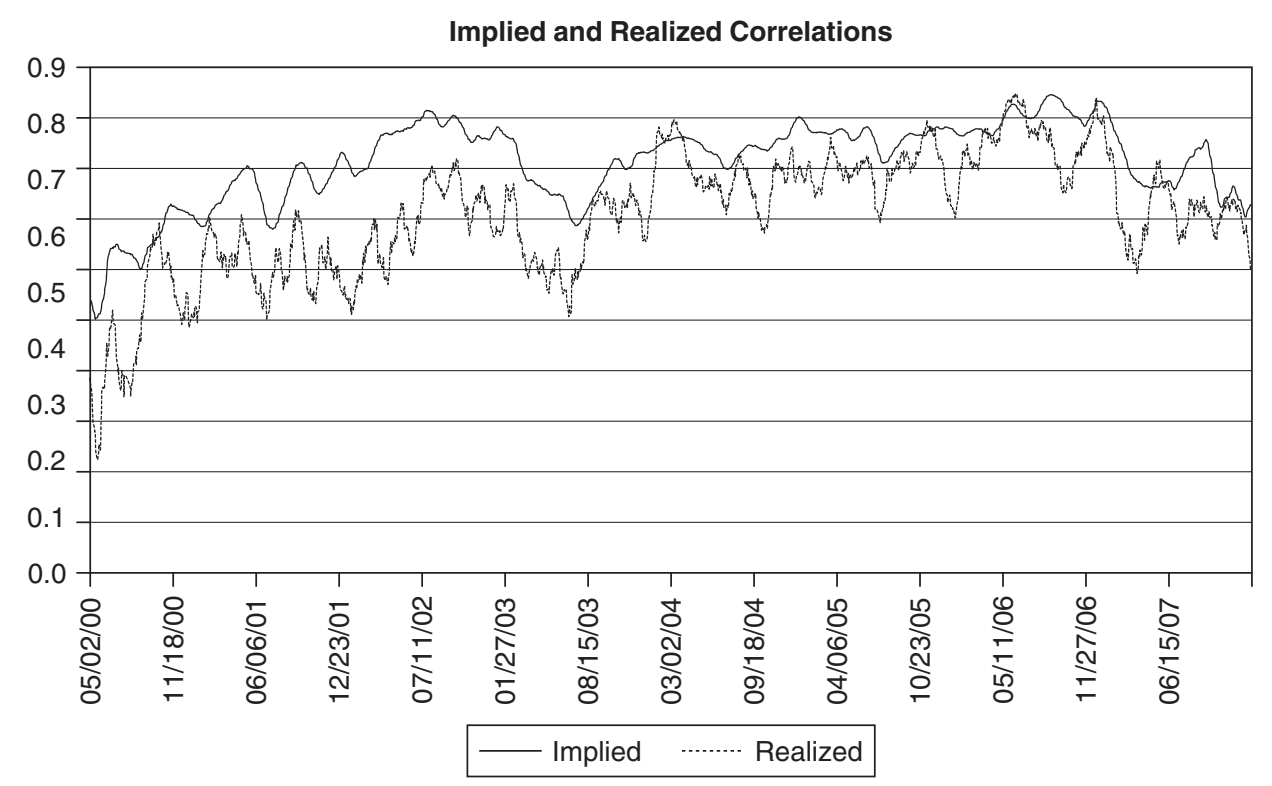

FIGURE 11

Time series of implied and realized correlation coefficients for the dollar/pound and dollar/euro rates from 2000 to 2007 . Each implied correlation is calculated from the three-month at-the-money implied volatilities of OTC options on the dollar/pound, dollar/euro, and euro/pound rates from May 2000 to December 2007. Each realized correlation is calculated from the 30-minute returns of OTC dollar/pound and dollar/euro rates during this period. All data are collected from Olsen and Associates. OTC, over-the-counter.

jumps; the jump proxy is $J_{t}=\max \left(\mathrm{RV}_{t}-\mathrm{BP}_{t}, 0\right)$ with $\mathrm{RV}_{t}$ and $\mathrm{BP}_{t}$ as the realized variance and the bipower variation of 30-minute cross-rate returns (Andersen, Bollerslev, \& Diebold, 2007; Barndorff-Nielsen \& Shephard, 2006). The euro and pound interest rates have approximately opposite coefficients in our best model; therefore, we specify the model as depending on the interest rate differential, namely $i_{€, t}-i_{€, t}$. We estimate the following regression model:

$d_{t}=\alpha+\beta_{1} d_{t-1}+\beta_{2} d_{t-2}+\beta_{3}\left(i_{€, t}-i_{£, t}\right)+\beta_{4} i_{\S, t}+\beta_{5} J_{t}+\beta_{6} I_{t}+\beta_{7}\left|r_{t}\right|+\varepsilon_{t}$

with a $\operatorname{GARCH}(1,1)$ specification for the residuals $\varepsilon_{t}$. As shown in Table VI, the most significant explanatory variable for the eight-year period from 2000 to 2007 is the interest rate differential, followed by the jump proxy variable. We conclude that the correlation differences have been higher when euro interest rates have been high relative to pound interest rates and/or there have been relatively extreme intraday returns, which are evidence of price jumps. We also find that the explanatory power of the models we have investigated is weak because $R^{2}$ is always less than $8 \%$. 
TABLE Vı

Determinants of the Correlation Risk Premium

\begin{tabular}{lrcrc}
\hline Coefficient & Estimate & Standard Error & t-Ratio & $p$-Value \\
\hline$\alpha$ & 0.1514 & 0.0183 & 8.2938 & 0.0000 \\
$\beta_{1}$ & 0.1256 & 0.0205 & 6.1255 & 0.0000 \\
$\beta_{2}$ & 0.0659 & 0.0210 & 3.1320 & 0.0017 \\
$\beta_{3}$ & 0.0409 & 0.0004 & 107.3063 & 0.0000 \\
$\beta_{4}$ & -0.0052 & 0.0023 & -2.2202 & 0.0264 \\
$\beta_{5}$ & 794.9835 & 70.3352 & 11.3028 & 0.0000 \\
$\beta_{6}$ & 0.0932 & 0.2484 & 0.3750 & 0.7077 \\
$\beta_{7}$ & 3.6760 & 1.1458 & 3.2082 & 0.0013 \\
Adjusted $R^{2}$ & 0.0769 & & & \\
\hline
\end{tabular}

The regression model estimated is

$$
d_{t}=\alpha+\beta_{1} d_{t-1}+\beta_{2} d_{t-2}+\beta_{3}\left(i_{\varepsilon, t}-i_{\varepsilon, t}\right)+\beta_{4} i_{s, t}+\beta_{5} J_{t}+\beta_{6} l_{t}+\beta_{7}\left|r_{t}\right|+\varepsilon_{t}
$$

with a $\operatorname{GARCH}(1,1)$ specification for the residuals $\varepsilon_{t}$. Here $d_{t}$ denotes the correlation difference on day $t$, defined as the threemonth ATM implied correlation minus the realized correlation calculated from 30-minute returns. Three-month interest rates for the dollar, pound, and euro are denoted by $i_{\$, t}, i_{\varepsilon, t}, i_{€ \in t}$ respectively. $i_{t}$ and $\left|r_{t}\right|$ are, respectively, the cross-rate implied volatility and the historical volatility proxied by the cross-rate absolute return. $J_{t}=\max \left(\mathrm{RV}_{t}-\mathrm{BP}_{t}, 0\right)$ is a proxy for cross-rate price jumps with $\mathrm{RV} \mathrm{V}_{t}$ and $\mathrm{BP}_{t}$ being the realized variance and bipower variation of 30 -minute cross-rate returns. ATM, at-the-money.

\section{CONCLUDING REMARKS}

This study illustrates practical methods for estimating cross-rate RNDs. We suppose that there is limited information available about the prices of crossrate options, which contrasts with an abundance of information about the prices of dollar-rate options. The theoretical cross-rate RND is derived from the bivariate RND for two dollar exchange rates. Cross-rate RNDs can be used to price any European contingent claim on cross rates and they also provide insight into market expectations.

Our methods make an assumption about the number of available implied volatilities for cross-rate options. We find that cross-rate option prices are sensitive to this number, which is assumed to be either one or zero. When there are no cross-rate option prices we have to rely on historical dollar exchange rates to quantify the dependence between the two dollar rates. The historical correlation estimates are positive and on average less than those implied by option prices.

Our methods use a copula function to provide an empirical approximation to the function defined by the bivariate RND of the dollar rates divided by the product of the marginal RNDs. The Frank, Gaussian, and Gumbel copulas provide satisfactory results, when we compare the cross-rate RNDs obtained from one cross-rate option price and many dollar-rate option prices with the densities that can be estimated from seven cross-rate option prices quoted by a 
particular bank. These comparisons show that the cross-rate RND can be estimated equally well from exchange-traded and OTC dollar-rate option prices.

Finally, we offer some advice to practitioners wishing to price cross-rate currency options. Dollar-rate marginal RNDs can be obtained by several methods and the selection of the method is probably not particularly important. Calculation of the bivariate RND of the dollar rates requires a specific copula function and this choice is important. The Gaussian copula is the only oneparameter copula function that is ranked highly in all of the comparisons we have made. The dependence parameter of the Gaussian copula is a critical number. The empirical analysis suggests that it is best to select this parameter within a risk-neutral framework; therefore, ideally practitioners should make use of the ATM cross-rate option price when they calculate the prices of other cross-rate options.

\section{APPENDIX A}

To simplify (4) into (5) it is necessary to simplify the function

$$
h(x)=\frac{\partial^{2}}{\partial x^{2}}\left[\int_{0}^{\infty} \int_{0}^{\infty} \max (y-x z, 0) f_{\$}(y, z) d y d z\right]
$$

First, let

$$
g(x)=\int_{0}^{\infty} \int_{0}^{\infty} \max (y-x z, 0) f_{\$}(y, z) d y d z=\int_{0}^{\infty}\left[\int_{x z}^{\infty}(y-x z) f_{\$}(y, z) d y\right] d z
$$

and replace $y$ by $t z$. Then $d y=z d t$ and $g(x)$ becomes

$$
g(x)=\int_{0}^{\infty} z^{2}\left[\int_{x}^{\infty}(t-x) f_{\$}(t z, z) d t\right] d z
$$

Second, differentiate the inner integral twice, obtaining

$$
\begin{aligned}
\frac{\partial}{\partial x} \int_{x}^{\infty}(t-x) f_{\$}(t z, z) d t & =-\int_{x}^{\infty} f_{\$}(t z, z) d t \quad \text { and } \\
\frac{\partial^{2}}{\partial x^{2}} \int_{x}^{\infty}(t-x) f_{\$}(t z, z) d t & =f_{\$}(x z, z) .
\end{aligned}
$$

Consequently, $h(x)$ simplifies to

$$
h(x)=\int_{0}^{\infty} z^{2} f_{\$}(x z, z) d z .
$$




\section{APPENDIX B}

The Gaussian copula is defined by

$$
C(u, v \mid \rho)=\int_{-\infty}^{s} \int_{-\infty}^{t} f(y, z \mid \rho) d y d z
$$

with $s=\Phi^{-1}(u)$ and $t=\Phi^{-1}(v)$; $\Phi$ is the standard normal c.d.f., and $f(\cdot \mid \rho)$ denotes the standard bivariate normal density with correlation $\rho$. The copula density is

$$
c(u, v \mid \rho)=\frac{1}{\sqrt{1-\rho^{2}}} \exp \left\{-\frac{1}{2\left(1-\rho^{2}\right)}\left[s^{2}+t^{2}-2 \rho s t\right]+\frac{1}{2}\left[s^{2}+t^{2}\right]\right\} .
$$

From Kepner, Harper, and Keith (1989), Spearman's $\rho$ for this density equals

$$
\rho_{S}=(6 / \pi) \arcsin (\rho / 2)
$$

The Gaussian copula generates a bivariate normal density if and only if the marginal densities are normal.

The Plackett copula is defined by a constant cross-product ratio:

$$
\frac{P(U \leq u, V \leq v) P(U>u, V>v)}{P(U \leq u, V>v) P(U>u, V \leq v)}=\theta
$$

with $\theta$ as a positive parameter. Consequently, with $\eta=\theta-1$,

$$
C(u, v \mid \theta)=\frac{1}{2} \eta^{-1}\left\{1+\eta(u+v)-\left[(1+\eta(u+v))^{2}-4 \theta \eta u v\right]^{1 / 2}\right\}
$$

and

$$
c(u, v \mid \theta)=\left[(1+\eta(u+v))^{2}-4 \theta \eta u v\right]^{-3 / 2} \theta[1+\eta(u+v-2 u v)] .
$$

The Frank, Clayton, and Gumbel copulas are all Archimedean copulas. They are defined by $C(u, v)=\varphi^{-1}(\varphi(u)+\varphi(v))$ for some continuous, strictly decreasing function $\varphi$ from $(0,1]$ to $[0, \infty)$ that has $\varphi(1)=0$.

For the Frank copula,

$$
\begin{gathered}
\varphi(t)=-\ln [(\exp (-\theta t)-1) /(\exp (-\theta)-1)], \theta \neq 0, \\
C(u, v \mid \theta)=-\frac{1}{\theta} \ln \left[1+\frac{\left(e^{-\theta u}-1\right)\left(e^{-\theta v}-1\right)}{e^{-\theta}-1}\right] \text { and } \\
c(u, v \mid \theta)=\frac{-\theta\left(e^{-\theta}-1\right) e^{-\theta(u+v)}}{\left[e^{-\theta}-1+\left(e^{-\theta u}-1\right)\left(e^{-\theta v}-1\right)\right]^{2}} .
\end{gathered}
$$


For the Clayton copula, with $\theta$ positive,

$$
\varphi(t)=\left(t^{-\theta}-1\right) / \theta, C(u, v \mid \theta)=\left(u^{-\theta}+v^{-\theta}-1\right)^{-1 / \theta}
$$

and

$$
c(u, v \mid \theta)=(1+\theta)(u v)^{-\theta-1}\left(u^{-\theta}+v^{-\theta}-1\right)^{-2-(1 / \theta)} .
$$

The Clayton copula can also be defined, by different equations, when $-1<$ $\theta<0$, but none of our estimates are within this range.

For the Gumbel copula,

$$
\varphi(t)=(-\ln t)^{\theta}, \theta \geq 1, C(u, v \mid \theta)=e^{-\left[(-\ln u)^{\theta}+(-\ln v)^{\theta}\right]^{1 / \theta}}
$$

and

$$
\begin{aligned}
c(u, v \mid \theta)= & C(u, v \mid \theta)(u v)^{-1} \frac{[(-\ln u)(-\ln v)]^{\theta-1}}{\left[(-\ln u)^{\theta}+(-\ln v)^{\theta}\right]^{2-1 / \theta}} \\
& \times\left\{\left[(-\ln u)^{\theta}+(-\ln v)^{\theta}\right]^{1 / \theta}+\theta-1\right\}
\end{aligned}
$$

\section{APPENDIX C}

RNDs are defined for an exchange rate $T$ years into the future when the spot rate for one unit of foreign currency is $S_{T}$ dollars. The dollar risk-free interest rate is $r$ and the forward rate at time zero for exchange at time $T$ is denoted by $F$; it equals the risk-neutral expectation of $S_{T}$. Interest rates are assumed to be nonstochastic so that $F$ is also a futures price. A general exercise price is represented by $X$, for a European call option that is written either on spot currency or on a futures contract that delivers at time $T$.

\section{The Generalized Beta Density}

Bookstaber and McDonald (1987) proposed the GB2 density for asset prices, with four positive parameters that define a parameter vector $\theta=(a, b, p, q)$. The RND for $S_{T}$ is

$$
f_{G B 2}(x \mid a, b, p, q)=\frac{a}{b^{a p} B(p, q)} \times \frac{x^{a p-1}}{\left[1+(x / b)^{a}\right]^{p+q}}, x>0
$$

where the function $B$ is defined in terms of the Gamma function by $B(p, q)=$ $\Gamma(p) \Gamma(q) / \Gamma(p+q)$. Call prices can be derived if $a q>1$, with the following result: 


$$
\begin{aligned}
C(X)= & F e^{-r T}\left[1-F_{\beta}\left(u(X, a, b) \mid p+a^{-1}, q-a^{-1}\right)\right] \\
& -X e^{-r T}\left[1-F_{\beta}(u(X, a, b) \mid p, q)\right]
\end{aligned}
$$

where $F_{\beta}$ is the incomplete beta function given by

$$
F_{\beta}(u \mid p, q)=\frac{1}{B(p, q)} \int_{0}^{u} t^{p-1}(1-t)^{q-1} d t \quad \text { and } \quad u(X, a, b)=\frac{(X / b)^{a}}{1+(X / b)^{a}} .
$$

Risk-neutrality constrains the mean of the density to be

$$
F=b B\left(p+a^{-1}, q-a^{-1}\right) / B(p, q) .
$$

\section{The Lognormal-Mixture Density}

The lognormal RND for $S_{T}$, with volatility $\sigma$, is the following density:

$$
\psi(x \mid F, \sigma, T)=\frac{1}{x \sigma \sqrt{2 \pi T}} \exp \left[-\frac{1}{2}\left(\frac{\ln (x / F)+(1 / 2) \sigma^{2} T}{\sigma \sqrt{T}}\right)^{2}\right] .
$$

The price of a call option is then given by Black's formula:

$$
C_{B}(F, T, X, r, \sigma)=F e^{-r T} \Phi\left(d_{1}\right)-X e^{-r T} \Phi\left(d_{2}\right)
$$

with $d_{1}=\left(\ln (F / X)+(1 / 2) \sigma^{2} T\right) /(\sigma \sqrt{T})$ and $d_{2}=d_{1}-\sigma \sqrt{T}$. A mixture of two lognormal densities for $S_{T}$ is defined by

$$
f_{\text {mix }}(x)=p \psi\left(x \mid F_{1}, \sigma_{1}, T\right)+(1-p) \psi\left(F_{2}, \sigma_{2}, T\right) .
$$

There are five non-negative parameters, $\theta=\left(F_{1}, F_{2}, \sigma_{1}, \sigma_{2}, p\right)$, with $0 \leq p \leq 1$. The mixture is risk-neutral when $F=p F_{1}+(1-p) F_{2}$. Call prices are simply a mixture of Black prices:

$$
C(X)=p C_{B}\left(F_{1}, T, X, r, \sigma_{1}\right)+(1-p) C_{B}\left(F_{2}, T, X, r, \sigma_{2}\right) .
$$

\section{The Lognormal-Polynomial Density}

Madan and Milne (1994) assume that the density of standardized returns $Z$ is the standard normal density $\phi(z)$ multiplied by a general function approximated by a polynomial. For a quartic approximation, the parameters are denoted by $\theta=\left(\mu, \sigma, b_{3}, b_{4}\right)$ such that $\log \left(F_{T}\right)-\log (F)$ has mean and variance, respectively, equal to $\mu T-\sigma^{2} T$ and $\sigma^{2} T$. The RND for $Z$ is

$$
f_{\mathrm{Z}}\left(z \mid \mu, \sigma, b_{3}, b_{4}\right)=\phi(z)\left[1+\frac{b_{3}}{\sqrt{6}}\left(z^{3}-3 z\right)+\frac{b_{4}}{\sqrt{24}}\left(z^{4}-6 z^{2}+3\right)\right]
$$

and the RND for $S_{T}$ is 


$$
f_{L P}\left(x \mid \mu, \sigma, b_{3}, b_{4}\right)=\frac{1}{x \sigma \sqrt{T}} f_{Z}\left(\frac{\ln (x)-\ln (F)-\mu T+0.5 \sigma^{2} T}{\sigma \sqrt{T}}\right)
$$

Risk-neutrality constrains the parameters to satisfy

$$
1+\frac{\beta^{3} b_{3}}{\sqrt{6}}+\frac{\beta^{4} b_{4}}{\sqrt{24}}=e^{-\mu T}
$$

with $\beta=\sigma \sqrt{T}$. The price of a call option is given by

$$
C(X)=e^{-r T}\left[a_{0}(X)+a_{3}(X) b_{3}+a_{4}(X) b_{4}\right]
$$

with $a_{0}(X)=F e^{\mu T} \Phi\left(D_{1}\right)-X \Phi\left(D_{1}-\sigma \sqrt{T}\right)$,

$$
\begin{aligned}
& \begin{aligned}
a_{3}(X) & =F \beta e^{\mu T}\left[\beta^{2} \Phi\left(D_{1}\right)+\left(2 \beta-D_{1}\right) \phi\left(D_{1}\right)\right] / \sqrt{6} \\
a_{4}(X) & =F \beta e^{\mu T}\left[\beta^{3} \Phi\left(D_{1}\right)+\left(3 \beta^{2}-3 \beta D_{1}+D_{1}^{2}-1\right) \phi\left(D_{1}\right)\right] / \sqrt{24} \\
\text { and } D_{1} & =\left(\ln (F / X)+\mu T+\frac{1}{2} \sigma^{2} T\right) /(\sigma \sqrt{T}) .
\end{aligned}
\end{aligned}
$$

\section{Parameter Estimation}

As is common in the RND literature, we estimate the parameters by minimizing the sum of squared pricing errors for a set of market call prices denoted by $c_{m}\left(X_{i}\right)$. When there are market prices available for $N$ distinct exercise prices, having a common expiry time $T$, we estimate $\theta$ by minimizing

$$
G(\theta)=\sum_{i=1}^{N}\left(c_{m}\left(X_{i}\right)-C\left(X_{i} \mid \theta\right)\right)^{2} .
$$

\section{BIBLIOGRAPHY}

Anagnou-Basioudis, I., Bedendo, M., Hodges, S. D., \& Tompkins, R. (2005). Forecasting accuracy of implied and GARCH-based probability density functions. Review of Futures Markets, 11, 41-66.

Andersen, T. G., Bollerslev, T., \& Diebold, F. X. (2007). Roughing it up: Including jump components in the measurement, modeling and forecasting of return volatility. Review of Economics and Statistics, 89, 701-720.

Andersen, T. G., Bollerslev, T., Diebold, F. X., \& Labys, P. (2001). The distribution of realized exchange rate volatility. Journal of the American Statistical Association, 96, 42-55.

Barndorff-Nielsen, O. E., \& Shephard, N. (2006). Econometrics of testing for jumps in financial economics using bipower variation. Journal of Financial Econometrics, $4,1-30$. 
Barone-Adesi, G., \& Whaley, R. E. (1987). Efficient analytic approximation of American option values. Journal of Finance, 42, 301-320.

Bartram, S. M., Taylor, S. J., \& Wang, Y. (2007). The Euro and European financial market dependence. Journal of Banking \& Finance, 31, 1461-1481.

Bennett, M. N., \& Kennedy, J. E. (2004). Quanto pricing with copulas. Journal of Derivatives, 12, 26-45.

Bikos, A. (2000). Bivariate FX PDFs: A sterling ERI application (working paper). Bank of England.

Bliss, R. R., \& Panigirtzoglou, N. (2002). Testing the stability of implied probability density functions. Journal of Banking \& Finance, 26, 381-422.

Bookstaber, R. M., \& McDonald, J. B. (1987). A general distribution for describing security price returns. Journal of Business, 60, 401-424.

Branger, N., \& Schlag, C. (2004). Why is the index smile so steep? Review of Finance, $8,109-127$.

Breeden, D. T., \& Litzenberger, R. H. (1978). Prices of state-contingent claims implicit in options prices. Journal of Business, 51, 621-651.

Campa, J. M., Chang, P. H., \& Reider, R. L. (1998). Implied exchange rate distributions: Evidence from OTC option markets. Journal of International Money and Finance, 17, 117-160.

Cherubini, U., \& Luciano, E. (2002). Bivariate option pricing with copulas. Applied Mathematical Finance, 9, 69-86.

Cherubini, U., Luciano, E., \& Vecchiato, W. (2004). Copula methods in finance. England: John Wiley \& Sons.

Driessen, J. J. A. G., Maenhout, P., \& Vilkov, G. (2009). The price of correlation risk: Evidence from equity options. Journal of Finance, 64, 1377-1406.

Hennessy, D. A., \& Lapan, H. (2002). The use of Archimedean copulas to model portfolio allocations. Mathematical Finance, 12, 143-154.

Jackwerth, J. C., \& Rubinstein, M. (1996). Recovering probability distributions from option prices. Journal of Finance, 51, 1611-1631.

Joe, H. (1997). Multivariate models and dependence concepts. London: Chapman \& Hall.

Jondeau, E., \& Rockinger, M. (2000). Reading the smile: The message conveyed by methods which infer risk neutral densities. Journal of International Money and Finance, 19, 885-915.

Jorion, P. (1995). Predicting volatility in the foreign exchange market. Journal of Finance, 50, 507-528.

Kepner, J. L., Harper, J. D., \& Keith, S. Z. (1989). A note on evaluating a certain orthant probability. The American Statistician, 43, 48-49.

Li, D. X. (2000). On default correlation: A copula function approach. Journal of Fixed Income, 9, 43-54.

Liu, X., Shackleton, M. B., Taylor, S. J., \& Xu, X. (2007). Closed-form transformations from risk-neutral to real-world distributions. Journal of Banking \& Finance, 31, 1501-1520.

Madan, D. B., \& Milne, F. (1994). Contingent claims valued and hedged by pricing and investing in a basis. Mathematical Finance, 4, 223-245.

Malz, A. M. (1996). Using option prices to estimate realignment probabilities in the European monetary system: The case of sterling-mark. Journal of International Money and Finance, 15, 717-748. 
Malz, A. M. (1997). Estimating the probability distribution of the future exchange rate from option prices. Journal of Derivatives, 5, 18-36.

Melick, W. R., \& Thomas, C. P. (1997). Recovering an asset's implied PDF from option prices: An application to crude oil during the Gulf crisis. Journal of Financial and Quantitative Analysis, 32, 91-115.

Nelsen, R. B. (1999). An introduction to copulas. New York: Springer.

Pong, S., Shackleton, M. B., Taylor, S. J., \& Xu, X. (2004). Forecasting currency volatility: A comparison of implied volatilities and AR(FI)MA models. Journal of Banking \& Finance, 28, 2541-2563.

Ritchey, R. J. (1990). Call option valuation for discrete normal mixtures. Journal of Financial Research, 13, 285-296.

Rosenberg, J. V. (2003). Non-parametric pricing of multivariate contingent claims. Journal of Derivatives, 10, 9-26.

Söderlind, P., \& Svensson, L. E. O. (1997). New techniques to extract market expectations from financial instruments. Journal of Monetary Economics, 40, 383-429.

Taylor, S. J. (2005). Asset price dynamics, volatility, and prediction. Princeton, NJ: Princeton University Press.

Xu, X., \& Taylor, S. J. (1994). The term structure of volatility implied by foreign exchange options. Journal of Financial and Quantitative Analysis, 29, 57-74. 